\title{
Parenteral glutamine supplementation in critical illness: a systematic review
}

\author{
Paul E Wischmeyer ${ }^{1 *}$, Rupinder Dhaliwal ${ }^{2}$, Michele McCall ${ }^{3}$, Thomas R Ziegler ${ }^{4}$ and Daren K Heyland ${ }^{2,5}$
}

\begin{abstract}
Introduction: The potential benefit of parenteral glutamine (GLN) supplementation has been one of the most commonly studied nutritional interventions in the critical care setting. The aim of this systematic review was to incorporate recent trials of traditional parenteral GLN supplementation in critical illness with previously existing data.
\end{abstract}

Methods: All randomized controlled trials of parenterally administered GLN in critically ill patients conducted from 1997 to 2013 were identified. Studies of enteral GLN only or combined enteral/parenteral GLN were excluded. Methodological quality of studies was scored and data was abstracted by independent reviewers.

Results: A total of 26 studies involving 2,484 patients examining only parenteral GLN supplementation of nutrition support were identified in ICU patients. Parenteral GLN supplementation was associated with a trend towards a reduction of overall mortality (relative risk (RR) $0.88,95 \%$ confidence interval $(C I) 0.75,1.03, P=0.10$ ) and a significant reduction in hospital mortality (RR $0.68,95 \% \mathrm{Cl} 0.51,0.90, P=0.008$ ). In addition, parenteral GLN was associated with a strong trend towards a reduction in infectious complications (RR 0.86, 95\% Cl $0.73,1.02, P=0.09$ ) and ICU length of stay (LOS) (WMD $-1.91,(95 \% \mathrm{Cl}-4.10,0.28, P=0.09)$ and significant reduction in hospital LOS (WMD -2.56 , $95 \% \mathrm{Cl}-4.71,-0.42, P=0.02$ ). In the subset of studies examining patients receiving parenteral nutrition (PN), parenteral GLN supplementation was associated with a trend towards reduced overall mortality (RR $0.84,95 \% \mathrm{Cl}$ $0.71,1.01, P=0.07)$.

Conclusions: Parenteral GLN supplementation given in conjunction with nutrition support continues to be associated with a significant reduction in hospital mortality and hospital LOS. Parenteral GLN supplementation as a component of nutrition support should continue to be considered to improve outcomes in critically ill patients.

\section{Introduction}

Glutamine (GLN) is the most abundant nonessential free amino acid [1] and has traditionally been classified as a nonessential amino acid able to be synthesized de novo in states of health. Glutamine is now commonly described as a conditionally essential amino acid, particularly in catabolic and stress states [2]. In catabolic states, large amounts of GLN are released from muscle tissue [3] as part of the body's conserved evolutionary response to stress. Previous explanations for the release of GLN in periods of stress include use as a fuel source for rapidly dividing cells, a precursor for synthesis of nucleic acids, and a role in renal acid buffering $[4,5]$. Recent data has

\footnotetext{
* Correspondence: Paul.Wischmeyer@ucdenver.edu

${ }^{1}$ Department of Anesthesiology, University of Colorado School of Medicine, 12700 East 19th Avenue, Aurora, CO 80045, USA

Full list of author information is available at the end of the article
}

revealed that following illness and injury, GLN plays a vital role in inducing cellular protection pathways, modulation of the inflammatory response, and prevention of organ injury [1]. Contrary to long-held beliefs, not all critically ill patients become markedly deficient in the first few days of ICU admission, although some will present with severe depletion, such as is consistently seen in burn patients [6-8]. Recent data has shown GLN becomes depleted in approximately 25 to $35 \%$ of ICU patients at admission to ICU [6-8]. Patients with more prolonged critical illness, and in some studies, those receiving parenteral nutrition $(\mathrm{PN})$ have shown show a greater level of depletion as described in a number of trials of PN-requiring ICU patients $[9,10]$. When GLN depletion is present at admission to ICU, it has been associated with increased mortality $[7,8]$. Clinical trials from the past 20 years demonstrated that GLN appears to reduce mortality, infectious

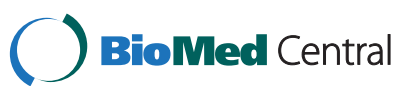


complications, and ICU/hospital length of stay (LOS) [1]. Most of these previous trials were conducted in a select group of patients with the following characteristics: 1) predominantly receiving complete nutrition support via $\mathrm{PN}, 2$ ) common exclusion of renal and liver failure based on glutamine product prescribing restrictions, 3) GLN supplementation of $\mathrm{PN}$ is often initiated later in the ICU stay (not at ICU admission during the shock phase), as PN is typically started later in the stay when enteral nutrition (EN) is not possible or meeting nutritional needs, 4) enrollment of oncology patients who may be at a greater risk for tumor-mediated GLN depletion, 5) use of GLN dose between $0.3 \mathrm{~g} / \mathrm{kg} /$ day to a maximum of $0.5 \mathrm{~g} . \mathrm{kg} /$ day given only via the intravenous route. This is summarized below:

Characteristics of traditional parenteral glutaminesupplementation trials

1. Enrolled patients requiring parenteral nutrition in most trials (85\% of published trials)

2. Glutamine given as a supplement to nutrition support - not as a separate pharmaconutrient independent of nutrition support

3. GLN supplementation given with the start of PN later in the ICU stay, often when EN was not possible or failing to meet nutritional needs. (Not typically at ICU admission)

4. Common exclusion of renal and liver failure based on glutamine product prescribing restrictions (See Table 1 for specific trial exclusion criteria)

5. Lower GLN doses ( 0.3 to $0.5 \mathrm{~g} / \mathrm{kg} /$ day)

6. Combined enteral and parenteral GLN not given

Recently, a new paradigm of pharmacological GLN administration was tested in a large multicenter clinical trial utilizing a combined enteral and intravenous dose of GLN, which averaged between 0.6 to $0.8 \mathrm{~g} / \mathrm{kg} /$ day independent of the administration of complete nutrition [6]. This study, the REDOXS study, also primarily enrolled patients who, as defined by the inclusion criteria, were required to be in multi-system organ failure within 24 hours of admission. Finally, renal failure and acute liver failure were, for the first time, not excluded and in fact, more than $30 \%$ of the patients in REDOXS presented with baseline acute renal failure. The results of this trial surprisingly showed contrary to many previous traditional PN-based GLN trials, that GLN supplementation was associated with an increase in mortality.

Thus, a key question to be answered is whether parenteral GLN administered as a supplement to complete nutrition support (for example via PN) is beneficial or harmful. This question is timely given the large number of $(n=11)$ of randomized clinical trials of GLN supplementation of PN published since 2009. Recently a number of meta- analysis have been published examining the use of GLN in burn injury, pancreatitis, and surgical and critical illness combined [11-14], however a systematic analysis focused on parenteral GLN supplementation in critical illness has not been performed. Further, these past meta-analysis have not incorporated all of the most recently available trial data. The aim of this current systematic literature review and meta-analysis is to focus on the question of whether the 'traditional' parenteral administration of GLN as part of nutrition support has an effect on relevant clinical outcomes in patients classified with critical illness (as opposed to elective surgery or only single diagnosis groups, such as burn injury).

\section{Methods}

\section{Study identification}

The following databases were searched for articles from 1980 until July 2013: EMBASE, MEDLINE, CINAHL and the Cochrane Controlled Trials Register and Database of Systematic Reviews. The literature search used broad search terms containing 'randomized', 'blind', 'clinical trial', 'nutrition', 'nutritional support' or 'dietary supplementation' or 'enteral nutrition' or 'parenteral nutrition' or 'parenteral nutrition solutions' and 'critical care' or 'critical illness' or 'intensive care units'. The results were then reviewed to identify articles using parenteral or intravenous GLN supplementation. A unique feature of this meta-analysis is that no language restrictions were placed on the searches. Personal files and reference lists of relevant review articles were also reviewed. As this was a systematic review no ethics board approval or patient consent was required.

\section{Study selection criteria}

We only included original studies if they met the following inclusion criteria: a) study design: randomized clinical trials, b) population: critically ill adult patients ( $>18$ years of age), defined as patients admitted to an ICU. When this was unclear, we considered a mortality rate higher than $5 \%$ (hospital mortality and if this was not reported we used ICU mortality or 28-day mortality) in the control group to be consistent with critical illness, c) intervention: parenteral GLN versus control (either isonitrogenous amino acid control) or placebo, d) study outcomes: must have included one of the following: mortality, ICU and hospital LOS, infectious complications, and other clinically important complications. Studies of enteral GLN only or combined enteral/parenteral GLN were excluded).

\section{Data abstraction}

Decisions about the inclusion of the articles were made in duplicate. All original studies were reviewed independently by two reviewers using a data abstraction form with a scoring system as shown in Table 2. An assessment of 
Table 1 Randomized studies evaluating glutamine (PN) in critically ill patients

\begin{tabular}{|c|c|c|c|c|c|c|c|c|c|c|}
\hline \multirow[t]{2}{*}{ Study } & \multirow[t]{2}{*}{ Population } & \multirow{2}{*}{$\begin{array}{l}\text { Exclusions for } \\
\text { renal/liver failure }\end{array}$} & \multirow{2}{*}{$\begin{array}{l}\text { Methods } \\
\text { (score) }\end{array}$} & \multirow{2}{*}{$\begin{array}{l}\text { Intervention dose of } \\
\text { glutamine } \mathrm{gm} / \mathrm{kg} / \mathrm{day}\end{array}$} & \multicolumn{2}{|c|}{ Mortality \# (\%)† } & \multicolumn{2}{|c|}{ Infections \# (\%)‡ } & \multicolumn{2}{|c|}{ Length of stay (days) } \\
\hline & & & & & Experimental & Control & Experimental & Control & Experimental & Control \\
\hline $\begin{array}{l}\text { 1) Griffiths } 1997 \\
\& 2002[28,29]\end{array}$ & $\begin{array}{l}\text { Single-center, } \\
\text { mixed ICU, } \\
\text { patients, } N=84\end{array}$ & Not defined & $\begin{array}{l}\text { C.Random: yes, } \\
\text { IT: yes, Blinding: } \\
\text { yes (11) }\end{array}$ & $\begin{array}{l}\text { PN, } 0.26 \text { IV glutamine + } \\
\text { PN vs. PN, isocaloric, } \\
\text { isonitrogenous }\end{array}$ & $\begin{array}{l}\text { Hospital } \\
18 / 42(43)\end{array}$ & $\begin{array}{l}\text { Hospital } \\
25 / 42(60)\end{array}$ & $28 / 42(67)$ & $26 / 42(62)$ & $\begin{array}{l}\text { ICU } 10.5 \\
(6-19)^{*}\end{array}$ & ICU $10.5(6-24)^{*}$ \\
\hline $\begin{array}{l}\text { 2) Powell-Tuck } \\
1999 \text { [36] }\end{array}$ & $\begin{array}{l}\text { Single-center, } \\
\text { mixed } \\
\text { ICU/hospital, } \\
\text { patients } \\
\mathrm{N}=168\end{array}$ & $\begin{array}{l}\text { Renal: creatinine } \\
>200 \mu \mathrm{mol} / / \text { Liver: if } \\
\text { failure resulted in a } \\
\text { PTR }>1.8 \text { or hepatic } \\
\text { encephalopathy }\end{array}$ & $\begin{array}{l}\text { C.Random: yes } \\
\text { ITT: yes Blinding: } \\
\text { yes (8) }\end{array}$ & $\begin{array}{l}\text { PN, } 0.26 \text { IV glutamine + } \\
\text { PN vs. PN, isocaloric, } \\
\text { isonitrogenous. }\end{array}$ & $\begin{array}{l}\text { Hospital } \\
14 / 83(17)\end{array}$ & $\begin{array}{l}\text { Hospital } \\
20 / 85(24)\end{array}$ & NR & NR & $\begin{array}{l}\text { Hospital } \\
43.4 \pm 34.1 \\
\quad(83)\end{array}$ & $\begin{array}{l}\text { Hospital } \\
48.9 \pm 38.4 \\
(85)\end{array}$ \\
\hline $\begin{array}{l}\text { 3) Wischmeyer } \\
2001 \text { [40] }\end{array}$ & $\begin{array}{l}\text { Single-center, } \\
\text { critically ill } \\
\text { burns } N=31\end{array}$ & $\begin{array}{l}\text { Renal: severe failure, } \\
\text { Liver: severe failure }\end{array}$ & $\begin{array}{l}\text { Random: not } \\
\text { sure ITT: no } \\
\text { Blinding yes (8) }\end{array}$ & $\begin{array}{l}\text { PN, } 0.57 \text { IV glutamine + } \\
\text { EN or EN + PN vs. } \\
\text { AAcids + PN or EN or } \\
\text { EN + PN, isocaloric, } \\
\text { Isonitrogenous }\end{array}$ & $\begin{array}{l}\text { Hospital } \\
1 / 12(8)\end{array}$ & $\begin{array}{l}\text { Hospital } \\
4 / 14(29)\end{array}$ & $7 / 12(58)$ & 9/14 (64) & $\begin{array}{l}\text { Hospital } \\
40 \pm 10 \\
(12)\end{array}$ & $\begin{array}{l}\text { Hospital } \\
40 \pm 9(14)\end{array}$ \\
\hline $\begin{array}{l}\text { 4) Fuentes- } \\
\text { Orozco } \\
2004 \text { [25] }\end{array}$ & $\begin{array}{l}\text { Single-center, } \\
\text { secondary } \\
\text { peritonitis } \\
\text { requiring TPN } \\
N=33\end{array}$ & $\begin{array}{c}\text { Renal: creatinine } \\
>180 \mu \mathrm{mol} / \mathrm{l} \text {. Liver: } \\
\text { bilirubin }>40 \mu \mathrm{mol} / \mathrm{l}, \\
\text { alanine aminotransferase } \\
>100 \mathrm{U} / \mathrm{l} \text { and aspartate } \\
\text { aminotransferase }>100 \mathrm{U} / \mathrm{l}\end{array}$ & $\begin{array}{l}\text { C.Random: yes, } \\
\text { ITT: yes. Blinding: } \\
\text { double (11) }\end{array}$ & $\begin{array}{l}\text { PN, } 0.27 \text { IV glutamine + } \\
\text { PN vs. PN, isocaloric, } \\
\text { isonitrogenous }\end{array}$ & $\begin{array}{l}\text { Hospital } \\
2 / 17(12)\end{array}$ & $\begin{array}{l}\text { Hospital } \\
3 / 16(19)\end{array}$ & 4/17 (23) & $12 / 16(75)$ & $\begin{array}{c}\text { ICU } 7.2 \pm 9.2 \\
\text { (17), Hospital, } \\
16.5 \pm 8.9 \\
(17)\end{array}$ & $\begin{array}{c}\text { ICU } \\
7.3 \pm 4.5(16) \\
\text { Hospital } \\
16.7 \pm 7(16)\end{array}$ \\
\hline $\begin{array}{l}\text { 5) Zhou } \\
2004[42]\end{array}$ & $\begin{array}{l}\text { Severe burns } \\
\qquad N=30\end{array}$ & $\begin{array}{l}\text { Renal: chronic disease. } \\
\text { Liver: chronic disease }\end{array}$ & $\begin{array}{l}\text { C.Random: yes. } \\
\text { ITT: yes. Blinding: } \\
\text { double (11) }\end{array}$ & $\begin{array}{l}\text { PN, } 0.35 \text { IV glutamine + } \\
\text { PN vs. PN, isocaloric, } \\
\text { isonitrogenous. }\end{array}$ & NR & NR & $3 / 15(20)$ & 4/15 (26) & $\begin{array}{l}\text { Hospital } \\
42 \pm 7.0 \\
(15)\end{array}$ & $\begin{array}{c}\text { Hospital } \\
46 \pm 6.6(15)\end{array}$ \\
\hline $\begin{array}{l}\text { 6) Xian-Li } \\
2004[30]\end{array}$ & $\begin{array}{l}\text { Single-center, } \\
\text { severe acute } \\
\text { pancreatitis, } \\
\quad N=69\end{array}$ & $\begin{array}{l}\text { Renal: renal dysfunction. } \\
\text { Liver: liver dysfunction }\end{array}$ & $\begin{array}{c}\text { C.Random: yes. } \\
\text { ITT: no Blinding: } \\
\text { no (5) }\end{array}$ & $\begin{array}{c}\text { PN, } 0.4 \text { IV glutamine }+ \\
\text { PN vs. PN }\end{array}$ & $\begin{array}{l}\text { Hospital } \\
0 / 20(0)\end{array}$ & $\begin{array}{l}\text { Hospital } \\
3 / 21(14)\end{array}$ & \# Compl 4 & \# Compl 11 & $\begin{array}{l}\text { Hospital } \\
25.3 \pm 7.6 \\
(20)\end{array}$ & $\begin{array}{c}\text { Hospital } \\
28.6 \pm 6.9(21)\end{array}$ \\
\hline \multirow[t]{2}{*}{$\begin{array}{l}\text { 7) Dechelotte } \\
2006 \text { [9] }\end{array}$} & $\begin{array}{l}\text { Multiple trauma, } \\
\text { surgery, sepsis, } \\
\text { pancreatitis from } \\
16 \text { ICUs N = } 114\end{array}$ & & & & & & & & & \\
\hline & $\begin{array}{l}\text { Renal: creatinine } \\
>250 \mu \text { mol/L. Liver: } \\
\text { Prothrombin } \\
\text { time }>23.7 \mathrm{sec}\end{array}$ & $\begin{array}{l}\text { C. Random: NR. } \\
\text { ITT: yes. Blinding: } \\
\text { double (NA) }\end{array}$ & $\begin{array}{c}\text { PN, } 0.35 \mathrm{IV} \\
\text { glutamine + PN } \\
\text { vs. PN, isocaloric, } \\
\text { isonitrogenous. }\end{array}$ & $\begin{array}{l}\text { Hospital 2/58 (3), } \\
\text { 6-month 16/58 (28) }\end{array}$ & $\begin{array}{l}\text { Hospital 2/56 } \\
\text { (3) 6-month } \\
9 / 56(16)\end{array}$ & $\begin{array}{l}\text { All 23/58 } \\
\text { (40) Pneumonia } \\
\text { 10/58 (17) }\end{array}$ & $\begin{array}{l}\text { All 32/56 (58), } \\
\text { Pneumonia } \\
19 / 56(34)\end{array}$ & $\begin{array}{c}\text { ICU } 12.5 \\
(1-430) \\
\text { Hospital } 30 \\
(1-560)\end{array}$ & $\begin{array}{l}\text { ICU } 11.5 \\
(3-121)_{1}^{*} \\
\text { Hospital } 26 \\
(4-407)^{*}\end{array}$ & \\
\hline $\begin{array}{l}\text { 8) Palmese } \\
2006 \text { [33] }\end{array}$ & $\begin{array}{l}\text { Single-center, } \\
\text { mixed ICU } \\
\quad \mathrm{N}=84\end{array}$ & $\begin{array}{l}\text { Renal: severe renal } \\
\text { disease. Liver: severe } \\
\text { hepatic disease }\end{array}$ & $\begin{array}{l}\text { C.Random: yes, } \\
\text { ITT: yes, Blinding: } \\
\text { single (10) }\end{array}$ & $\begin{array}{c}\text { PN, } 0.14 \text { IV glutamine + } \\
\text { EN with FOS } \\
\text { vs. EN without FOS }\end{array}$ & ICU 6/42 (14) & $\begin{array}{c}\text { ICU } \\
8 / 42(19)\end{array}$ & $\begin{array}{l}\text { All 13/42 (31), } \\
\text { Pneumonia } \\
\text { 2/42 (5) }\end{array}$ & $\begin{array}{l}\text { All 21/42 (50), } \\
\text { Pneumonia } \\
\text { 6/42 (14) }\end{array}$ & $\begin{array}{l}\text { ICU } 12 \pm 4.6 \\
\quad(42)\end{array}$ & $\begin{array}{c}\text { ICU } 13 \pm 3.4 \\
(42)\end{array}$ \\
\hline $\begin{array}{l}\text { 9) Tian } \\
2006 \text { [38] }\end{array}$ & $\begin{array}{l}\text { Single-center, } \\
\text { MODS N }=40\end{array}$ & Not defined & $\begin{array}{l}\text { C.Random: not } \\
\text { sure, ITT: yes, } \\
\text { Blinding: } \\
\text { none (6) }\end{array}$ & $\begin{array}{l}\text { PN, } 0.27 \text { IV } \\
\text { glutamine vs. PN }\end{array}$ & $\begin{array}{l}\text { Unspecified } \\
2 / 20(10)\end{array}$ & $\begin{array}{l}\text { Unspecified } \\
5 / 20(25)\end{array}$ & NR & NR & NR & NR \\
\hline
\end{tabular}


Table 1 Randomized studies evaluating glutamine (PN) in critically ill patients (Continued)

\begin{tabular}{|c|c|c|c|c|c|c|c|c|c|c|}
\hline $\begin{array}{l}\text { 10) Sahin } \\
2007[37]\end{array}$ & $\begin{array}{l}\text { Single-center, } \\
\text { acute pancreatitis } \\
\qquad N=40\end{array}$ & Not defined & $\begin{array}{l}\text { C.Random: not } \\
\text { sure, ITT: yes, } \\
\text { Blinding: } \\
\text { single (9) }\end{array}$ & $\begin{array}{l}\text { PN, } 0.3 \text { IV glutamine + } \\
\text { PN vs. PN, isocaloric, } \\
\text { isonitorgenous. }\end{array}$ & $\begin{array}{l}\text { Hospital } \\
2 / 20(10)\end{array}$ & $\begin{array}{l}\text { Hospital } \\
6 / 20(30)\end{array}$ & NR & NR & $\begin{array}{l}\text { Hospital } \\
14.2 \pm 4.4 \\
(20)\end{array}$ & $\begin{array}{c}\text { Hospital } \\
16.4 \pm 3.9(20)\end{array}$ \\
\hline $\begin{array}{l}\text { 11) Zhang } \\
2007 \text { [43] }\end{array}$ & $\begin{array}{c}\text { Emergency and } \\
\text { neurosurgical ICU, } \\
\text { pts requiring } \\
\text { PN for }>7 \text { days, } \\
N=44\end{array}$ & Not defined & $\begin{array}{c}\text { C.Random: } \\
\text { not sure, ITT: } \\
\text { yes, Blinding: } \\
\text { no (6) }\end{array}$ & $\begin{array}{l}\text { EN and PN, IV } \\
\text { glutamine } \\
0.4 \mathrm{~g} / \mathrm{kg} / \text { day } \\
\text { vs. EN and } \\
\text { PN alone }\end{array}$ & NR & NR & NR & NR & $\begin{array}{c}I C U \\
11.73 \pm 6.57 \\
(22)\end{array}$ & $\begin{array}{c}\text { ICU } \\
13.39 \pm 5.08(22)\end{array}$ \\
\hline $\begin{array}{l}\text { 12) Cai } \\
2008[21]\end{array}$ & $\begin{array}{l}\text { Single-center, } \\
\text { elderly, severe } \\
\text { sepsis } N=110\end{array}$ & $\begin{array}{l}\text { Renal: creatinine } \\
>220 \mu \mathrm{mol} / \mathrm{L} \text { and/or } \\
\text { dialysis. Liver: bilirubin } \\
>43 \mu \mathrm{mol} / \mathrm{L} \text { and/or } \\
\text { history of chronic } \\
\text { liver disease }\end{array}$ & $\begin{array}{l}\text { C.Random: not } \\
\text { sure, ITT: yes, } \\
\text { Blinding: } \\
\text { no (10) }\end{array}$ & $\begin{array}{l}\text { PN, } 0.19 \mathrm{IV} \\
\text { glutamine Patients } \\
\text { received PN } \\
\text { or EN + PN } \\
\text { isocaloric, } \\
\text { isonitrogenous }\end{array}$ & $\begin{array}{l}\text { 28-day } \\
17 / 55 \text { (31) }\end{array}$ & $\begin{array}{l}\text { 28-day } \\
\text { 20/55 (36) }\end{array}$ & NR & NR & $\begin{array}{c}\text { ICU } \\
22.1 \pm 4.9 \\
(55)\end{array}$ & $\begin{array}{c}\text { ICU } \\
23.8 \pm 5.1(55)\end{array}$ \\
\hline $\begin{array}{l}\text { 13) Duska } \\
2008[15]^{\partial}\end{array}$ & $\begin{array}{l}\text { Single-center, } \\
\text { trauma } N=30\end{array}$ & None & $\begin{array}{l}\text { C.Random: not } \\
\text { sure, ITT: yes, } \\
\text { Blinding: } \\
\text { single (8) }\end{array}$ & $\begin{array}{c}\text { PN, } 0.3 \mathrm{IV} \\
\text { glutamine + PN vs. } \\
\text { normal saline + } \\
\text { supplemental PN, } \\
\text { isocaloric, } \\
\text { isonitrogenous }\end{array}$ & $\begin{array}{c}\text { ICU } \\
2 / 10(20)\end{array}$ & ICU 0/10 (0) & NR & NR & $\begin{array}{l}\text { ICU } 23 \\
\text { (median) }\end{array}$ & $\begin{array}{c}\text { ICU } 24 \\
\text { (median) }\end{array}$ \\
\hline $\begin{array}{l}\text { 14) Estivariz } \\
2008 \text { [24] }\end{array}$ & $\begin{array}{l}\text { Single-center, } \\
\text { pancreatic and } \\
\text { non pancreatic } \\
\text { surgery } N=63\end{array}$ & $\begin{array}{l}\text { Renal: evolving acute } \\
\text { renal failure or dialysis. } \\
\text { Liver: total bili }>4 \mathrm{mg} / \mathrm{dL} \\
\text { or }>5 \text { fold elevation in } \\
\text { serum transaminase } \\
\text { concentrations }\end{array}$ & $\begin{array}{l}\text { C.Random: not } \\
\text { sure, ITT: no**, } \\
\text { Blinding: } \\
\text { double (9) }\end{array}$ & $\begin{array}{c}\text { PN, } 0.5 \text { IV } \\
\text { glutamine + PN } \\
\text { vs. PN isocaloric, } \\
\text { isonitrogenous }\end{array}$ & $\begin{array}{l}\text { Hospital } \\
1 / 32(3)\end{array}$ & $\begin{array}{l}\text { Hospital } \\
6 / 31(19)\end{array}$ & $\begin{array}{l}\text { Pneumonia } \\
\text { 13/30 (43) }\end{array}$ & $\begin{array}{l}\text { Pneumonia } \\
16 / 29(55)\end{array}$ & $\begin{array}{c}\text { ICU } \\
12 \pm 2(32) \\
\text { Hospital } \\
20 \pm 2(32)\end{array}$ & $\begin{array}{c}\text { ICU } \\
23 \pm 6(31) \\
\text { Hospital } \\
30 \pm 6(31)\end{array}$ \\
\hline $\begin{array}{l}\text { 17) Perez- } \\
\text { Barcena } \\
2008 \text { [35] }\end{array}$ & $\begin{array}{l}\text { Single-center, } \\
\text { mixed ICU } N=30\end{array}$ & Not defined & $\begin{array}{l}\text { C.Random: } \\
\text { not sure, ITT: } \\
\text { yes, Blinding: } \\
\text { single (10) }\end{array}$ & $\begin{array}{c}\text { PN, } 0.35 \text { IV } \\
\text { glutamine + PN } \\
\text { vs. PN isocaloric, } \\
\text { isonitrogenous }\end{array}$ & $\begin{array}{l}\text { Hospital } \\
3 / 15 \text { (20) }\end{array}$ & $\begin{array}{l}\text { Hospital } \\
0 / 15(0)\end{array}$ & 11/15 (73) & 13/15 (87) & $\begin{array}{c}\text { ICU } \\
22.9 \pm 20.6 \\
(15), \text { Hospital, } \\
35.5 \pm 33.6 \\
\text { (15) }\end{array}$ & $\begin{array}{c}\text { ICU } 20.5 \pm 16.0 \\
\text { (15) Hospital } \\
42.9 \pm 28.8(15)\end{array}$ \\
\hline $\begin{array}{l}\text { 18) Ozgultekin } \\
2008 \text { [32] }\end{array}$ & $\begin{array}{l}\text { Single-center, } \\
\text { CHI \& GCS pts, } \\
\text { ventilated, } \\
\text { sedated, mean } \\
\text { APACHE II } \\
18-19 \mathrm{~N}=60\end{array}$ & Not defined & $\begin{array}{l}\text { C.Random: not } \\
\text { sure ITT: no, } \\
\text { Blinding: } \\
\text { none (4) }\end{array}$ & $\begin{array}{l}\text { EN, } 0.2-0.4 \mathrm{~g} / \mathrm{kg} / \mathrm{d} \\
\text { IV glutamine vs. EN }\end{array}$ & $\begin{array}{l}\text { 30-day } \\
12 / 20(60)\end{array}$ & $\begin{array}{l}\text { 30-day } \\
12 / 20(60)\end{array}$ & NR & NR & $\begin{array}{c}\text { ICU } \\
11.8 \pm 5.9 \\
(20)\end{array}$ & $\begin{array}{c}\text { ICU } 17.3 \pm 16.4 \\
(20)\end{array}$ \\
\hline $\begin{array}{l}\text { 19) Yang } \\
2008 \text { [41] }\end{array}$ & $\begin{array}{l}\text { Single-center, } \\
\text { severe } \\
\text { pancreatitis } \\
N=61\end{array}$ & Not defined & $\begin{array}{l}\text { C.Random: not } \\
\text { sure, ITT: no } \\
\text { Blinding: } \\
\text { single (4) }\end{array}$ & $\begin{array}{l}\text { PN, IV glutamine } \\
\text { (dose unknown) } \\
\text { vs. PN, saline }\end{array}$ & $\begin{array}{l}\text { Hospital } \\
1 / 25(4)\end{array}$ & $\begin{array}{l}\text { Hospital } \\
3 / 25(12)\end{array}$ & NR & NR & $\begin{array}{l}\text { Hospital } \\
13.48 \pm 1.42 \\
\quad(25)\end{array}$ & $\begin{array}{l}\text { Hospital } \\
15.18 \pm 1.14 \\
(25)\end{array}$ \\
\hline \multirow[t]{2}{*}{$\begin{array}{l}\text { 20) Eroglu } \\
2009[23]\end{array}$} & $\begin{array}{l}\text { Single-center, } \\
\text { severe trauma, } \\
\text { ISS }>20 \mathrm{~N}=40\end{array}$ & $\begin{array}{l}\text { Renal: on renal } \\
\text { replacement } \\
\text { therapy, } \\
\text { Liver: none }\end{array}$ & $\begin{array}{l}\text { C.Random: } \\
\text { yes, IT: yes, } \\
\text { Blinding: } \\
\text { double (12) }\end{array}$ & $\begin{array}{c}\mathrm{EN}, 0.5 \mathrm{~g} / \mathrm{kg} / \mathrm{d} \text { IV } \\
\text { glutamine vs. EN, } \\
\text { saline }\end{array}$ & ICU 1/20 (5) & ICU 1/20 (5) & $\begin{array}{l}\text { Overall } 8 / 20 \\
\text { (40) VAP } \\
1 / 20(5)\end{array}$ & $\begin{array}{c}\text { Overall } \\
10 / 20(50) \\
\text { VAP } 1 / 20(5)\end{array}$ & $\begin{array}{c}I C U \\
14 \pm 2(20)\end{array}$ & ICU $15 \pm 2(20)$ \\
\hline & & Not defined & & & & & & & & \\
\hline
\end{tabular}


Table 1 Randomized studies evaluating glutamine (PN) in critically ill patients (Continued)

\begin{tabular}{|c|c|c|c|c|c|c|c|c|c|c|}
\hline $\begin{array}{l}\text { 21) Perez- } \\
\text { Barcena } \\
2010[34]\end{array}$ & $\begin{array}{c}\text { Single-center, } \\
\text { trauma pt ISS } \\
>12 \text {, requires PN } \\
\text { based on ASPEN } \\
N=43\end{array}$ & & $\begin{array}{l}\text { C.Random: not } \\
\text { sure, IT: yes, } \\
\text { Blinding: } \\
\text { single (6) }\end{array}$ & $\begin{array}{l}\mathrm{PN}, 0.35 \mathrm{~g} / \mathrm{kg} / \mathrm{d} \text { IV } \\
\text { glutamine vs. PN }\end{array}$ & $\begin{array}{l}\text { ICU 4/23 (17), } \\
\text { Hospital } \\
\text { 0/23 (0) }\end{array}$ & $\begin{array}{c}\text { ICU 2/20 (10), } \\
\text { Hospital } \\
1 / 20(5)\end{array}$ & $\begin{array}{l}\text { Pneumonia } \\
11 / 23 \text { (48) }\end{array}$ & $\begin{array}{l}\text { Pneumonia } \\
8 / 20(40)\end{array}$ & $\begin{array}{c}\text { ICU 21 } \\
(17-25)_{1}^{*} \\
\text { Hospital } \\
31(19-42)^{*}\end{array}$ & $\begin{array}{c}\text { ICU } 21(14-47)^{*} \\
\text { Hospital } \\
40(24-80)^{*}\end{array}$ \\
\hline $\begin{array}{l}\text { 22) Andrews } \\
2011 \text { [20] }\end{array}$ & $\begin{array}{c}\text { Multi-center, } \\
\text { critically ill adults, } \\
25 \% \text { medical pts, } \\
\text { from } 10 \text { centres. } \\
N=502\end{array}$ & $\begin{array}{l}\text { Renal: estimated GFR } \\
<10 \mathrm{ml} / \mathrm{min} \text { and not } \\
\text { receiving renal } \\
\text { replacement } \\
\text { therapy. Liver: none }\end{array}$ & $\begin{array}{l}\text { C.Random: yes, } \\
\text { ITT: yes, Blinding: } \\
\text { double (13) }\end{array}$ & $\begin{array}{c}\text { PN, } 0.2-0.4 \mathrm{~g} / \mathrm{kg} / \text { day } \\
\text { (20.2 g/day } \mathrm{x} 7 \text { days) } \\
\text { IV glutamine } \\
\text { vs.PN isocaloric, } \\
\text { isonitrogenous }\end{array}$ & $\begin{array}{l}\text { ICU 88/250 } \\
\text { (35), 6-month } \\
115 / 250(46)\end{array}$ & $\begin{array}{c}\text { ICU 80/252 } \\
\text { (32), 6-month } \\
106 / 252(42)\end{array}$ & $134 / 250(54)$ & 131/252 (52) & $\begin{array}{c}\text { ICU 15 } \\
(7.9-28.4)^{*}, \\
\text { Hospital } \\
32.5 \\
(14.7-55.6)^{*}\end{array}$ & $\begin{array}{c}\text { ICU 13.4 } \\
(8.2-23.9)^{*}, \\
\text { Hospital } \\
28.2 \\
(15.1-52.4)^{*}\end{array}$ \\
\hline $\begin{array}{l}\text { 23) Cekmen } \\
2011 \text { [22] }\end{array}$ & $\begin{array}{c}\text { Single-center, } \\
\text { mixed surgical ICU, } \\
\text { ISS }>10, \text { APACHE II } \\
>10 \mathrm{~N}=30\end{array}$ & $\begin{array}{l}\text { Renal: significant renal } \\
\text { dysfunction (evolving acute } \\
\text { renal failure or on dialysis). } \\
\text { Liver: significant hepatic } \\
\text { dysfunction (total bilirubin } \\
>4.0 \mathrm{mg} / \mathrm{dL} \text { or more than } \\
\text { fivefold elevation in serum } \\
\text { transaminase concentrations) }\end{array}$ & $\begin{array}{l}\text { C.Random: yes, } \\
\text { ITT: yes, Blinding: } \\
\text { double (10) }\end{array}$ & $\begin{array}{l}\text { PN, } 0.5 \mathrm{~g} / \mathrm{kg} \text { IV } \\
\text { glutamine vs. PN }\end{array}$ & $\begin{array}{c}\text { ICU } \\
\text { (presumed) } \\
3 / 15(20)\end{array}$ & $\begin{array}{l}\text { ICU } \\
\text { (presumed) } \\
6 / 15 \text { (40) }\end{array}$ & NR & NR & $\begin{array}{c}\text { ICU } \\
19.2 \pm 12 \\
(15)\end{array}$ & $\begin{array}{c}\text { ICU } \\
27.4 \pm 12(15)\end{array}$ \\
\hline $\begin{array}{l}\text { 24) Grau } \\
2011[27]\end{array}$ & $\begin{array}{l}\text { Multi-center, } \\
\text { mechanically } \\
\text { ventilated, } \\
\text { APACHE II >12, } \\
\text { need TPN } \\
\quad \mathrm{N}=127\end{array}$ & $\begin{array}{l}\text { Renal: chronic renal failure } \\
\text { requiring dialysis, acute renal } \\
\text { failure not treated with } \\
\text { hemofiltration or } \\
\text { hemodialysis [plasmatic } \\
\text { creatinine }>2.5 \mathrm{mg} / \mathrm{dL} \text { ]. Liver: } \\
\text { hepatic failure with hepatic } \\
\text { encephalopathy or portal } \\
\text { hypertension }\end{array}$ & $\begin{array}{l}\text { C.Random: not } \\
\text { sure, ITT: yes } \\
\text { Blinding: } \\
\text { double (11) }\end{array}$ & $\begin{array}{l}\text { PN, } 0.5 \mathrm{~g} / \mathrm{kg} \text { IV } \\
\text { glutamine vs. PN }\end{array}$ & $\begin{array}{l}\text { ICU 9/59 (15), } \\
\text { 6-month } \\
16 / 59(27)\end{array}$ & $\begin{array}{c}\text { ICU 13/68 (19), } \\
\text { 6-month 23/68 } \\
\text { (34) }\end{array}$ & $\begin{array}{l}\text { All 24/59 (41), } \\
\text { Surgical, } \\
13 / 59(22), \\
\text { Pneu (\#/1000 } \\
\text { vent days), } \\
13.5, \# \text { i } \\
\text { nfect/pt } 1.5\end{array}$ & $\begin{array}{l}\text { All } 31 / 68(46) \text {, } \\
\text { Surgical, } \\
17 / 68 \text { (25), } \\
\text { Pneu (\#/1000 } \\
\text { vent days), } \\
27.2, \# \\
\text { infect/pt } 2.4\end{array}$ & $\begin{array}{l}\text { ICU } 12(7-22)^{*} \text {, } \\
\text { Hospital 35 } \\
(23-56)^{*}\end{array}$ & $\begin{array}{c}\text { ICU } \\
12(7-24)^{*}, \\
\text { Hospital, } \\
31(20-58)^{*}\end{array}$ \\
\hline $\begin{array}{l}\text { 25) Wernerman } \\
2011 \text { [39] }\end{array}$ & $\begin{array}{l}\text { Multi-center, } \\
\text { mixed ICU, } \\
\text { APACHE II >10 } \\
\quad \mathrm{N}=413\end{array}$ & Not defined & $\begin{array}{l}\text { C.Random: yes, } \\
\text { ITT: yes Blinding: } \\
\text { double (11) }\end{array}$ & $\begin{array}{c}\text { EN or PN, } 0.28 \\
\text { g/kg/day IV glutamine } \\
\text { vs. EN or PN, } \\
\text { normal saline IV }\end{array}$ & $\begin{array}{c}\text { ICU 8/205 (4) } \\
28-\text { day } \\
14 / 205 \text { (7) }\end{array}$ & $\begin{array}{c}\text { ICU } 11 / 208(5) \\
28 \text {-day } 20 / 208 \\
(10)\end{array}$ & NR & NR & NR & NR \\
\hline \multirow[t]{2}{*}{$\begin{array}{l}\text { 26) Ziegler } \\
2012 \text { [44] }\end{array}$} & $\begin{array}{l}\text { Multi-center, } \\
\qquad \mathrm{N}=150\end{array}$ & $\begin{array}{l}\text { Renal: history of chronic } \\
\text { renal failure requiring } \\
\text { dialysis, or significant renal } \\
\text { dysfunction (creatinine } \\
>2.5 \mathrm{mg} / \mathrm{dL} \text { and is not } \\
\text { receiving continuous renal } \\
\text { replacement therapy) or } \\
\text { requiring acute hemodialysis } \\
\text { postoperatively. Liver: current } \\
\text { encephalopathy or known } \\
\text { history of cirrhosis or total } \\
\text { bilirubin } \geq 10.0 \mathrm{mg} / \mathrm{dL}\end{array}$ & $\begin{array}{l}\text { C.Random: yes } \\
\text { ITT: yes, Blinding: } \\
\text { double (12) }\end{array}$ & $\begin{array}{l}\mathrm{PN}, 0.5 \mathrm{gm} / \mathrm{kg} / \text { day IV } \\
\text { Vs. PN, isocaloric. } \\
\text { Isonitrogenous. }\end{array}$ & $\begin{array}{l}\text { Hospital, } \\
11 / 75 \text { (15) }\end{array}$ & $\begin{array}{l}\text { Hospital } \\
13 / 75 \text { (17) }\end{array}$ & $\begin{array}{l}\text { Any 33/75 } \\
\quad(44) \text {. } \\
\text { Pneumonia, } \\
\text { 10/75 (13) }\end{array}$ & & & \\
\hline & $\begin{array}{c}\text { Any, 24/75 (32) } \\
\text { Pneumonia, } \\
\text { 12/75 (16) }\end{array}$ & $\begin{array}{l}\text { ICU } 17.5 \pm 14.6(75) \\
\text { Hospital } 33.6 \pm 28(75)\end{array}$ & $\begin{array}{c}\text { ICU } 13.6 \pm 10 \\
\text { (75). Hospital } \\
29.7 \pm 20.7(75)\end{array}$ & & & & & & & \\
\hline
\end{tabular}

${ }^{\dagger}$ Hospital mortality unless stated otherwise; ${ }^{\ddagger}$ number of patients with infections unless stated otherwise; ${ }^{*}$ data for length of stay is in median and ranges; ${ }^{* *}$ data for mortality is ITT, infections is non-ITT; ${ }^{2}$ data from growth hormone group not shown here. \pm ( ), mean \pm standard deviation (number); Compl, complications; ICU, intensive care unit; Infec/pt, infections per patient; C.Random, concealed randomization median (range); Pneu, pneumonia; PN, parenteral nutrition; ITT, intention to treat; EN, enteral nutrition; TPN, total parenteral nutrition; NR, not reported; FOS, fructooligosaccharides; MODS, multiple organ dysfunction syndrome; CHI, closed head injury; GCS, Glasgow Coma Scale; APACHE II, Acute Physiology and Chronic Health Evaluation II; ISS, injury severity score; Vent days, ventilator days; VAP, ventilator-associated pneumonia; ASPEN, American Society for Parenteral and Enteral Nutrition; GFR, glomerular filtration rate; NA, not applicable. 
Table 2 Randomized trial quality scoring system

\begin{tabular}{|c|c|c|c|}
\hline & & Score & \\
\hline & 0 & 1 & 2 \\
\hline Randomization & $\ldots$ & Not concealed or not sure & Concealed randomization \\
\hline Analysis & Other & $\ldots$ & Intention-to-treat \\
\hline Blinding & Not blinded & Single-blind & Double-blinded \\
\hline Patient selection & Selected patients or unable to tell & Consecutive eligible patients & $\ldots$. \\
\hline Comparability of groups at baseline & No or not sure & Yes & $\ldots$ \\
\hline Extent of follow-up & $<100 \%$ & $100 \%$ & $\ldots$ \\
\hline Treatment protocol & Poorly described & Reproducibly described & $\ldots$ \\
\hline Co-interventions & Not described & Described but not equal or not sure & Well described and all equal \\
\hline Outcomes & Not described & Partially described & Objectively defined \\
\hline
\end{tabular}

the criteria for inclusion, details on the patient population, intervention and control/placebo and clinical outcomes were made as per earlier publications [15]. The methodological quality of individual trials was also assessed according to a) whether randomization was concealed, b) blinding, c) analysis was based on the intention-to-treat (ITT) principle, d) patient selection, e) comparability of groups at baseline, f) extent of follow-up, g) description of treatment protocol and co-interventions, and h) definition of clinical outcomes. Each individual study was scored from 1 to 14 (Table 2). Disagreement in the individual scores of each of the categories was resolved by consensus between both reviewers. We attempted to contact the authors of included studies and requested additional information not contained in published articles.

\section{Data synthesis}

The primary outcome of the systematic review was overall mortality. From all studies, we combined hospital mortality where reported (specified or assumed to be hospital if not specified). If hospital mortality was not reported, we used ICU mortality or, if ICU mortality was not reported, we used 28-day mortality. Secondary outcomes included infection, ventilator-associated pneumonia (VAP), and ICU and hospital LOS. We used definitions of infections as defined by the authors in their original articles. We combined data from all trials to estimate the pooled risk ratio (RR) with $95 \%$ confidence intervals (CIs) for mortality, infectious complications, VAP and overall weighted mean difference (WMD) with 95\% CIs for LOS data. Pooled RRs were calculated using the MantelHaenszel estimator and WMDs were estimated by the inverse variance approach. The random effects model of Der Simonian and Laird was used to estimate variances for the Mantel-Haenszel and inverse variance estimators [16]. All analyses, except the test for asymmetry, were conducted using Review Manager (RevMan) 5.1 software. (The Nordic Cochrane Centre, The Cochrane Collaboration, Copenhagen, Denmark, 2011) [17].
When possible, studies were aggregated on an ITT basis (Table 2). The presence of heterogeneity was tested by a weighted Mantel-Haenszel chi-square test and quantified by the $\mathrm{I}^{2}$ statistic as implemented in RevMan $5.1[17,18]$. The possibility of publication bias was assessed by generating funnel plots and testing asymmetry of outcomes using methods proposed by Rucker and colleagues [19]. We considered $P<0.05$ to be statistically significant and $P<0.20$ as the indicator of trend.

\section{Subgroup analyses}

We utilized predefined subgroup analysis to assess a number of possible influences on the effect of parenteral GLN supplementation on clinical outcomes. We first examined the effect of parenteral GLN supplementation of $\mathrm{PN}$ versus EN alone. As trials examining EN alone consistently deliver significantly less total overall energy and protein delivery, we hypothesized that parenteral GLN may have a different effect when complete nutrition support was being given via EN alone or as a component of PN delivery. We next assessed the effect of trial quality on outcome as it is often hypothesized trials of lower methodological quality tend to yield more positive clinical signals for the therapy being tested when compared to trials of higher methodological quality. Utilizing our trial scoring tool, we designated trials with a methodological score of $>8$ as a 'high-quality trial' for the purposes of this review. Finally, it has been postulated that single-center trials may yield a greater chance of a positive clinical signal versus more rigorous multi-center trials [16], thus we examined the effect of these two types of trials on our clinical outcomes.

\section{Results}

\section{Study identification and selection}

The literature search yielded 58 potentially eligible randomized controlled trials of which 26 were included in our systematic review. As shown in Table S1 in Additional file 1, a total of 32 studies were excluded for these reasons: 
1) patients not considered to be critically ill $(\mathrm{n}=18), 2)$ no clinical outcomes $(n=5), 3)$ were duplicate studies or subgroups of included studies $(\mathrm{n}=3), 4)$ crossover design studies $(n=2), 5)$ varying doses of $\operatorname{GLN}(n=1), 6)$ a trial of combined enteral and parenteral GLN $(n=1), 7)$ questionably low dosage of GLN (0.002 g/kg/day) ( $\mathrm{n}=1$, see Table S1 in Additional file 1) and 8) one trial reported data from a subgroup of its total study population [10], see Table S1 in Additional file 1).

Thus, we included 26 studies of parenteral GLN supplementation performed in ICU patients with diagnosis ranging from pancreatitis, trauma, burns to sepsis described in Table $1[9,15,20-44]$. While in the majority of the studies, the intervention and control groups received GLN-free parenteral nutrition/amino acids, in four studies, patients received only EN as the sole source of nutrition support [23,31-33].

\section{Meta-analyses of primary and secondary outcomes Effect of GLN supplementation on mortality}

When the 24 studies that reported on mortality (Figure 1) were aggregated, IV GLN supplementation was associated with a trend towards a reduction in overall mortality (RR 0.88, 95\% CI 0.75, 1.03, $P=0.10$, heterogeneity $\mathrm{I}^{2}=0 \%$ ).
In the 13 studies (Figure 2) that reported hospital mortality, a significant reduction in hospital mortality was seen when they were aggregated (RR $0.68,95 \% \mathrm{CI} 0.51,0.90, P=0.008$, heterogeneity $\mathrm{I}^{2}=0 \%$ ).

\section{Effect of GLN supplementation on infectious complications, ICU length of stay and hospital length of stay}

When the 12 studies (Figure 3), which reported infectious complications, were aggregated, GLN supplementation was associated with a trend towards a reduction in infectious complications (RR 0.86, 95\% CI 0.73, 1.02, $P=0.09$, heterogeneity $\left.\mathrm{I}^{2}=43 \%\right)$. When the six studies that reported VAP (Figure 4) were aggregated, GLN supplementation was associated with a trend towards a reduction in pneumonia (RR $0.76,95 \%$ CI $0.56,1.04$, $P=0.09$, heterogeneity $\mathrm{I}^{2}=0 \%$ ).

When the 11 studies that reported ICU length of stay as a mean \pm standard deviation were aggregated (Figure 5), GLN supplementation was associated with a trend to reduction in ICU LOS (WMD -1.91, 95\% CI $-4.10,-0.28, P=0.09$, heterogeneity $\left.\mathrm{I}^{2}=90 \%\right)$. Finally, when the 11 studies reporting on hospital LOS were aggregated (Figure 6), GLN supplementation was associated with a significant reduction in hospital LOS

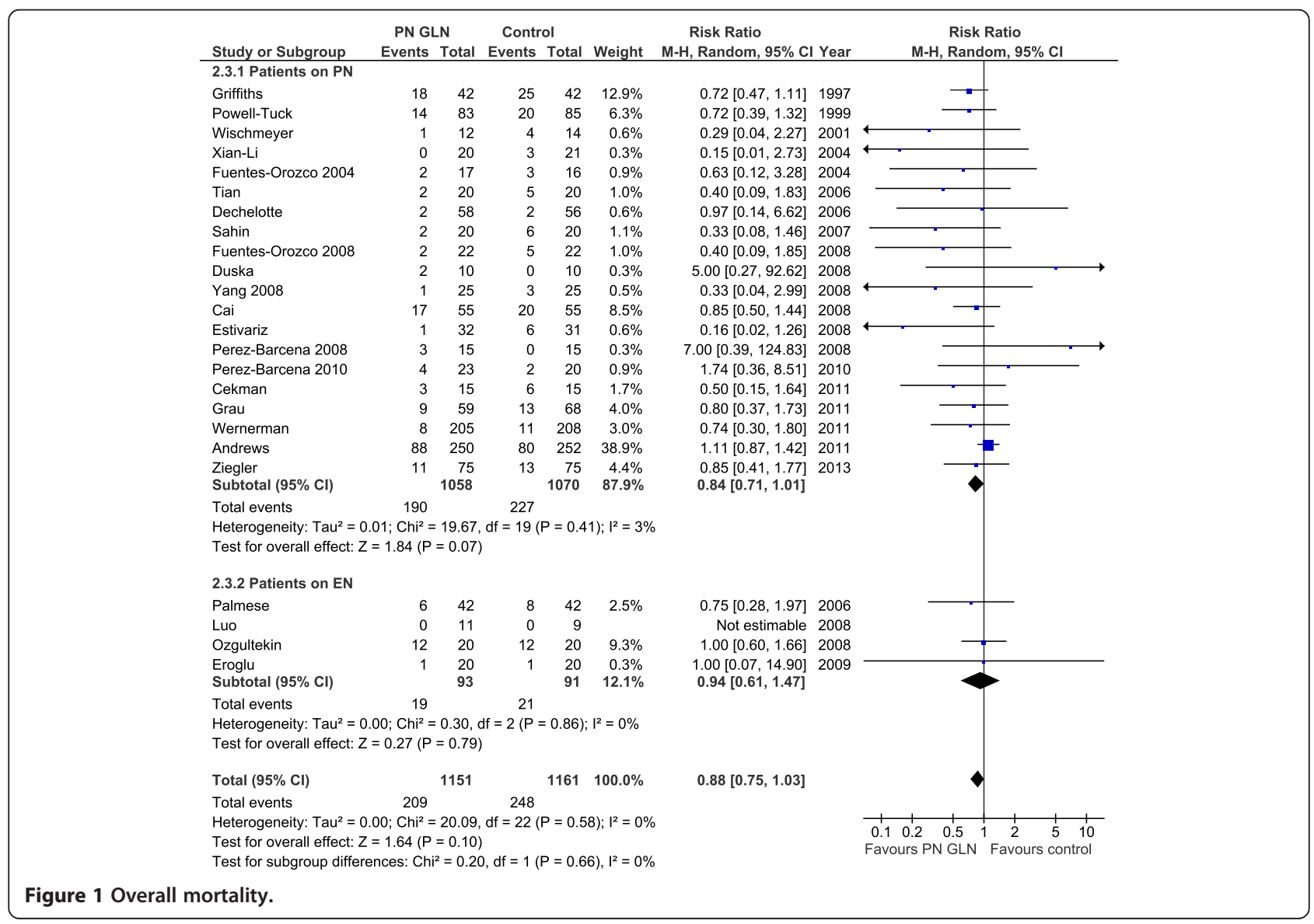




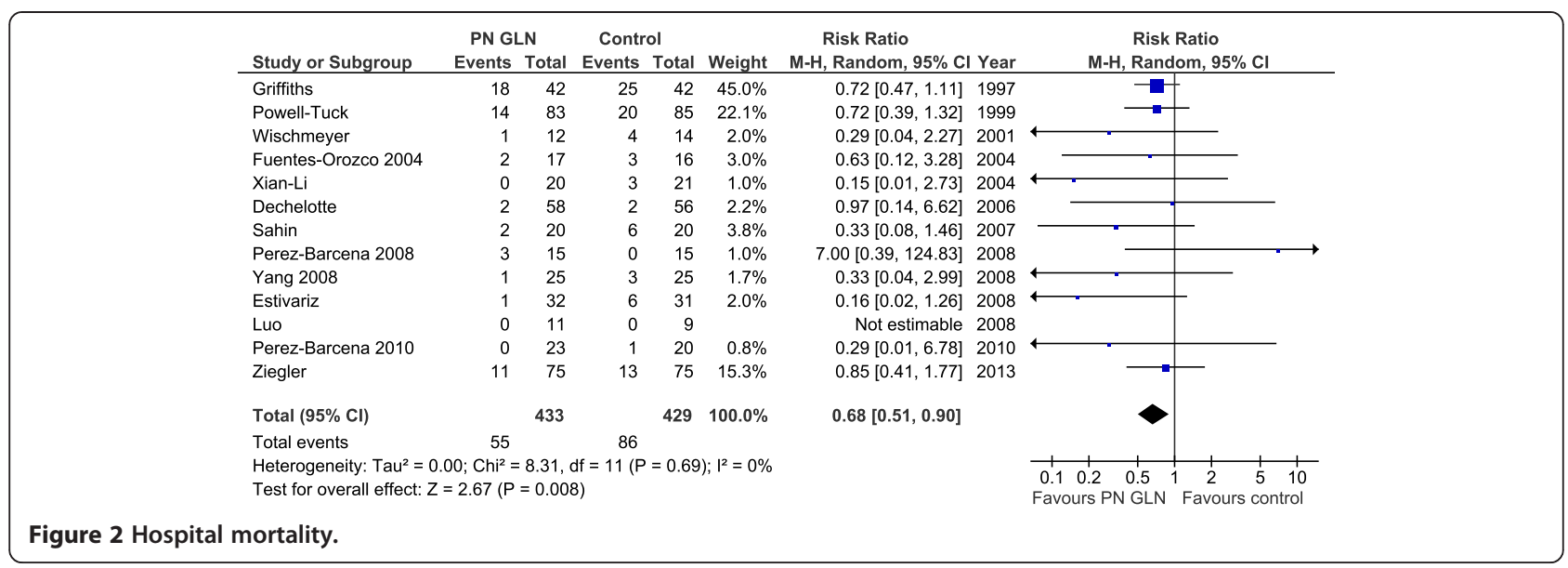

(WMD $-2.56,95 \% \mathrm{CI}-4.71,-0.42, P=0.02$, heterogeneity $\mathrm{I}^{2}=63 \%$ ).

\section{Subgroup analysis}

Results across all these subgroups are reported in Table 3. Overall tests for significance between subgroups revealed no statistically significant differences between any individual subgroups.

\section{Effect of parenteral GLN supplementation of PN versus EN alone}

Examining the effect of GLN supplementation of PN versus EN alone, the test for subgroup differences in overall mortality outcomes was not significant $(P=0.66)$. In the studies in which patients received intravenous (IV) GLN plus PN, GLN supplementation was associated with a trend towards a reduction in overall mortality (RR
$0.84,95 \%$ CI $0.71,1.01, P=0.07$, heterogeneity $\mathrm{I}^{2}=3 \%$; Figure 1, Table 3). Only four studies reported on mortality for GLN supplementation of EN. When the studies in which patients only received IV GLN and EN [23,31-33] were aggregated, GLN supplementation had no effect on overall mortality (RR 0.94, 95\% CI 0.61, $1.47, P=0.79$, heterogeneity $\mathrm{I}^{2}=0 \%$; Figure 1 , Table 3 ). In the subgroup of studies in which patients received IV GLN plus PN, GLN supplementation had no effect on infectious complications (RR 0.89, 95\% CI 0.74, 1.06, $P=0.20$, heterogeneity $\mathrm{I}^{2}=48 \%$; Figure 3 , Table 3 ). However, for the subgroup of studies in which patients received IV GLN and were on EN alone [23,33], GLN supplementation was associated with a trend towards a reduction in infectious complications (RR 0.68, 95\% CI $0.45,1.05, P=0.08$, heterogeneity $\mathrm{I}^{2}=0 \%$; Figure 3 , Table 3 ). The test for subgroup differences in infectious

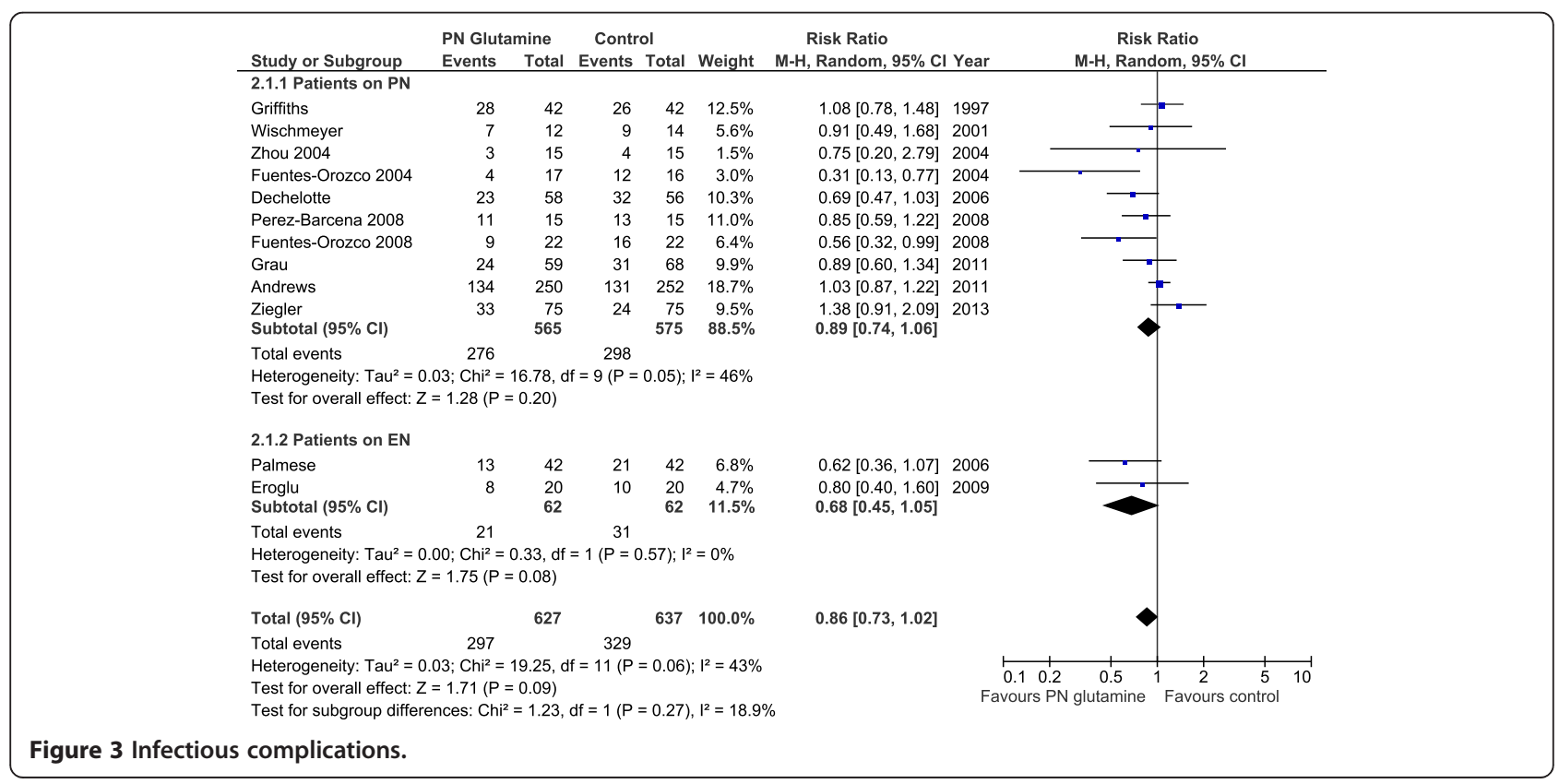


morbidity overall in EN alone versus PN studies was not significant $(P=0.27)$.

For the subgroup of studies in which patients received IV GLN plus PN, GLN supplementation was not associated with a reduction in ICU LOS. Similarly no effect on ICU LOS was observed in patients receiving only EN that received IV GLN [23,31-33]. The test for subgroup differences in ICU LOS overall was not significant $(P=0.42)$. None of the studies in which patients only received EN reported on hospital LOS and therefore no subgroup analyses were done.

\section{Effect of study quality on outcomes}

Higher quality trials (methodological score of $>8$ ) showed a stronger statistical trend towards reduced mortality than trials of lower quality $(P=0.12$ versus $P=0.49$ respectively), although the effect size was similar between the groups (Table 3). The overall test for subgroup difference was not significant for these subgroups $(P=0.79)$.

A statistically significant effect for GLN reducing hospital mortality was only seen in the higher quality trials, although there were a smaller number of patients enrolled in the lower quality trials and this reduced the statistical power of the signal (Table 3). Again, overall tests for significance did not reveal statistically significant differences between these subgroups $(P=0.21)$. There were insufficient numbers of trials reporting on infectious outcomes and LOS data in the low- and high-quality categories to allow for these comparisons to be made.

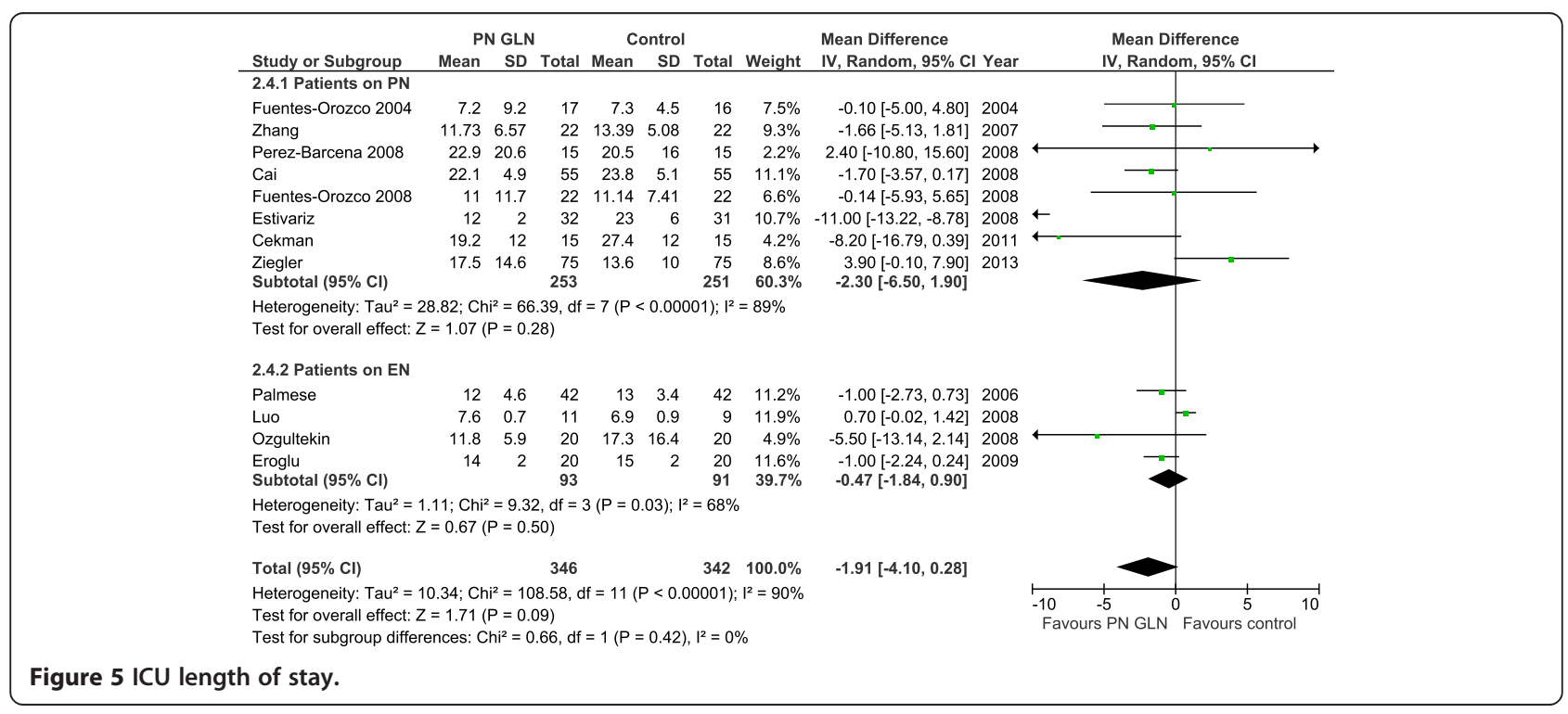




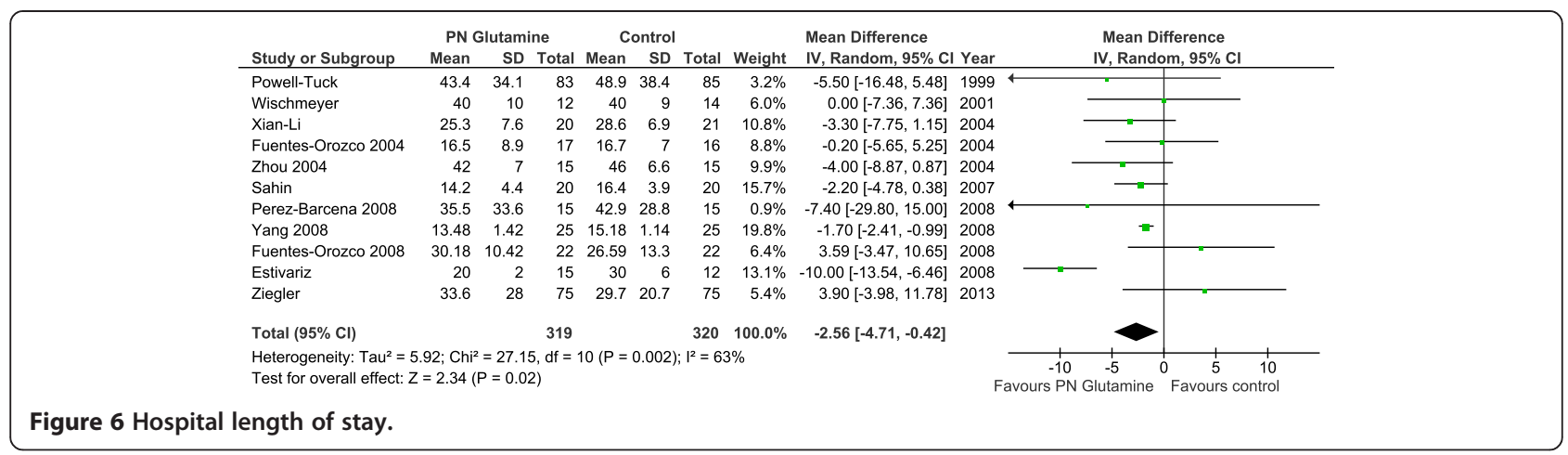

Role of multi-center versus single-center trials on outcomes The role of multi-center versus single center trials revealed that only the single center trials demonstrated a significant effect of GLN on overall and hospital mortality and infectious outcomes (Table 3). Only the multicenter trials demonstrated a significant benefit of GLN on the incidence of VAP (Table 3). Overall tests for significance did not reveal statistically significant differences between these subgroups. ( $P=0.45$ for mortality and $P=0.16$ for infectious morbidity, and $P=0.34$ for VAP).

\section{Risk of bias across studies}

Funnel plots for all outcomes were created to assess for publication bias as shown in Figure 7. The test of asymmetry was not found to be significant for any of the endpoints including overall mortality $(P=0.57)$, hospital mortality $(P=0.86)$, infectious complications $(P=0.05)$ ICU LOS (0.87), or hospital LOS (0.69).

\section{Discussion}

Meta-analysis of the initial randomized controlled trials of parenteral GLN supplementation suggested outcome benefits in the six early trials examining critical illness [45]. Since that time an additional 22 randomized clinical trials have been performed in over 2,000 patients receiving GLN as a component of complete nutrition support, primarily given as PN (85\% of trials). In addition, we have a much greater understanding of the potential beneficial mechanisms of GLN in critical illness as summarized in recent reviews $[1,46]$.

Early trials of GLN were primarily focused on patients receiving full nutrition support primarily by $\mathrm{PN}$ at doses falling within the approved prescribing indications for dose in commercially available GLN preparations. Further, as shown in Table 1, patients in these traditional trials were commonly excluded from enrollment if they had pre-existing renal or liver failure. These traditional nutritionally oriented (or non-pharmaconutrition based) GLN supplementation trials have shown a consistent reduction of mortality and benefit on other outcomes as suggested by the 2009 update of the Canadian Critical Care Nutrition Guidelines [47]. However, given the publication of 11 new randomized controlled trials examining the traditional parenteral use of GLN as a component of nutrition support (predominantly PN) since 2009, a new systematic review is indicated.

This need for a new systematic review is further driven by the results of the REDOXS trial, a 1,200-patient, 40-center randomized controlled trial of 'pharmacologically dosed' parenteral and enteral GLN (approximately 0.6 to $0.8 \mathrm{~g} / \mathrm{kg} /$ day) factorialized with antioxidant supplementation [6]. This trial was distinct from any of the previously published parenteral GLN trials supplementing PN or EN in ICU patients as GLN (and a cocktail of antioxidants) were administered independent of any concomitant nutrition support. In contrast to the design of studies reported in this analysis, the nutritional delivery of energy and protein in the REDOXS trial averaged less than 50\% of that prescribed for the patient, and thus was quite insufficient in meeting patient needs. In addition, all patients in the REDOXS trial had documented multi-system organ failure at enrollment, which is a common exclusion criteria in the trials reported in this analysis using parenteral GLN as a supplement to nutrition support. Further, more than $30 \%$ of REDOXS patients were found to have baseline renal failure at admission, which was a very common exclusion criteria in the trials reported in the analysis (Table 1). As stated, this is a key difference of REDOXS from all other GLN trials.

Thus, given the many differences in fundamental trial design between REDOXS and traditional GLN supplementation trials, the REDOXS trial has not been included in this systematic review focused on GLN supplementation of nutrition support. However, the publication of the REDOXS trial necessitates a close examination of the many new GLN supplementation trials published recently to examine if there has been a change in the fundamental signal of benefit of GLN supplementation of PN seen in historical trials in the recent era of critical care practice. This was the primary aim of this systematic review. 
Table 3 Subgroup analyses

\begin{tabular}{|c|c|c|c|c|c|c|c|c|c|c|c|c|}
\hline \multirow[b]{2}{*}{$\begin{array}{l}\text { Subgroup } \\
\text { analysis }\end{array}$} & \multicolumn{2}{|c|}{ Overall mortality } & \multirow[b]{2}{*}{$\begin{array}{l}\text { Effect on overall } \\
\text { mortality } \\
{[\mathrm{RR}(95 \% \mathrm{Cl}), p]}\end{array}$} & \multirow[b]{2}{*}{$\begin{array}{l}\text { Test for } \\
\text { subgroup } \\
\text { differences }\end{array}$} & \multicolumn{2}{|c|}{ Hospital mortality } & \multirow[b]{2}{*}{$\begin{array}{l}\text { Effect on } \\
\text { hospital mortality } \\
\text { [RR }(95 \% \mathrm{Cl}), p]\end{array}$} & \multirow[b]{2}{*}{$\begin{array}{l}\text { Test for } \\
\text { subgroup differences }\end{array}$} & \multicolumn{3}{|c|}{ Infectious complications } & \multirow[b]{2}{*}{$\begin{array}{l}\text { Test for } \\
\text { subgroup } \\
\text { differences }\end{array}$} \\
\hline & $\begin{array}{l}\text { Number } \\
\text { of trials }\end{array}$ & $\begin{array}{l}\text { Number } \\
\text { of patients }\end{array}$ & & & $\begin{array}{l}\text { Number } \\
\text { of trials }\end{array}$ & $\begin{array}{l}\text { Number } \\
\text { of patients }\end{array}$ & & & $\begin{array}{l}\text { Number } \\
\text { of trials }\end{array}$ & $\begin{array}{l}\text { Number } \\
\text { of patients }\end{array}$ & $\begin{array}{l}\text { Effect on infectious } \\
\text { complications } \\
\text { [RR }(95 \% \mathrm{Cl}), P]\end{array}$ & \\
\hline \multicolumn{13}{|l|}{ Study Quality } \\
\hline $\begin{array}{l}\text { Low quality } \\
(<8)\end{array}$ & 5 & 214 & $\begin{array}{l}0.81(0.44,1.48) \\
P=0.49\end{array}$ & $P=0.79$ & 3 & 134 & $\begin{array}{l}0.26(0.06,1.19) \\
P=0.08\end{array}$ & $P=0.21$ & NA & & & \\
\hline $\begin{array}{l}\text { High quality } \\
(\geq 8)\end{array}$ & 19 & 2103 & $\begin{array}{l}0.88(0.74,1.04) \\
P=0.12\end{array}$ & & 10 & 733 & $\begin{array}{l}0.70(0.52,0.94) \\
P=0.02\end{array}$ & & & & & \\
\hline \multicolumn{13}{|l|}{$\begin{array}{l}\text { Number of } \\
\text { sites }\end{array}$} \\
\hline $\begin{array}{l}\text { Single } \\
\text { center }\end{array}$ & 19 & 1011 & $\begin{array}{l}0.75(0.60,0.93) \\
P=0.009\end{array}$ & $P=0.04$ & 11 & 603 & $\begin{array}{l}0.64(0.47,0.88) \\
P=0.006\end{array}$ & $P=0.45$ & 8 & 371 & $\begin{array}{l}0.77(0.60,0.98) \\
P=0.03\end{array}$ & $P=0.16$ \\
\hline Multi-center & 5 & 1306 & $\begin{array}{l}1.03(0.83,1.28) \\
P=0.79\end{array}$ & & 2 & 264 & $\begin{array}{l}0.86(0.43,1.71) \\
P=0.67\end{array}$ & & 4 & 893 & $\begin{array}{l}0.97(0.77,1.23) \\
P=0.83\end{array}$ & \\
\hline \multicolumn{13}{|l|}{ PN vs. EN } \\
\hline $\begin{array}{l}\text { Patients on } \\
\text { PN }\end{array}$ & 20 & 2128 & $\begin{array}{l}0.84(0.71,1.01) \\
P=0.07\end{array}$ & $P=0.66$ & NA & & & & 10 & 1140 & $\begin{array}{l}0.89(0.74,1.06) \\
P=0.20\end{array}$ & $P=0.27$ \\
\hline $\begin{array}{l}\text { Patients on } \\
\text { EN }\end{array}$ & 4 & 184 & $\begin{array}{l}0.94(0.61,1.47) \\
P=0.79\end{array}$ & & & & & & 2 & 124 & $\begin{array}{l}0.68(0.45,1.05) \\
P=0.08\end{array}$ & \\
\hline
\end{tabular}


Table 3 Subgroup analyses (Continued)

\begin{tabular}{|c|c|c|c|c|c|c|c|c|}
\hline \multirow[b]{2}{*}{$\begin{array}{l}\text { Subgroup } \\
\text { analysis }\end{array}$} & \multicolumn{2}{|l|}{ ICU LOS } & \multirow[b]{2}{*}{$\begin{array}{l}\text { Effect on ICU LOS } \\
\text { [WMD }(95 \% \mathrm{Cl}), P]\end{array}$} & \multirow[b]{2}{*}{$\begin{array}{l}\text { Test for subgroup } \\
\text { differences }\end{array}$} & \multicolumn{2}{|l|}{ VAP } & \multirow[b]{2}{*}{$\begin{array}{l}\text { Effect on VAP } \\
\text { [RR }(95 \% \mathrm{Cl}), P]\end{array}$} & \multirow[b]{2}{*}{$\begin{array}{l}\text { Test for subgroup } \\
\text { differences }\end{array}$} \\
\hline & $\begin{array}{l}\text { Number } \\
\text { of trials }\end{array}$ & $\begin{array}{l}\text { Number } \\
\text { of patients }\end{array}$ & & & $\begin{array}{l}\text { Number } \\
\text { of trials }\end{array}$ & $\begin{array}{l}\text { Number } \\
\text { of patients }\end{array}$ & & \\
\hline \multicolumn{9}{|l|}{ Study Quality } \\
\hline Low quality $(<8)$ & NA & & & & NA & & & \\
\hline \multicolumn{9}{|l|}{ High quality ( $\geq 8$ ) } \\
\hline \multicolumn{9}{|l|}{ Number of sites } \\
\hline Single center & NA & & & & 4 & 226 & $\begin{array}{l}0.86(0.58,1.28) \\
P=0.45\end{array}$ & $P=0.34$ \\
\hline Multi-center & & & & & 2 & 264 & $\begin{array}{l}0.63(0.38,1.04) \\
P=0.07\end{array}$ & \\
\hline \multicolumn{9}{|l|}{$P N$ vs. EN } \\
\hline Patients on PN & 8 & 354 & $-2.30(-6.50,1.90) P=0.28$ & $P=0.42$ & 4 & 366 & $\begin{array}{l}0.79(0.57,1.09) \\
P=0.16\end{array}$ & $P=0.40$ \\
\hline Patients on EN & 3 & 184 & $-0.47(-1.84,0.90) P=0.50$ & & 2 & 124 & $\begin{array}{l}0.44(0.11,1.67) \\
P=0.23\end{array}$ & \\
\hline
\end{tabular}

not applicable. 

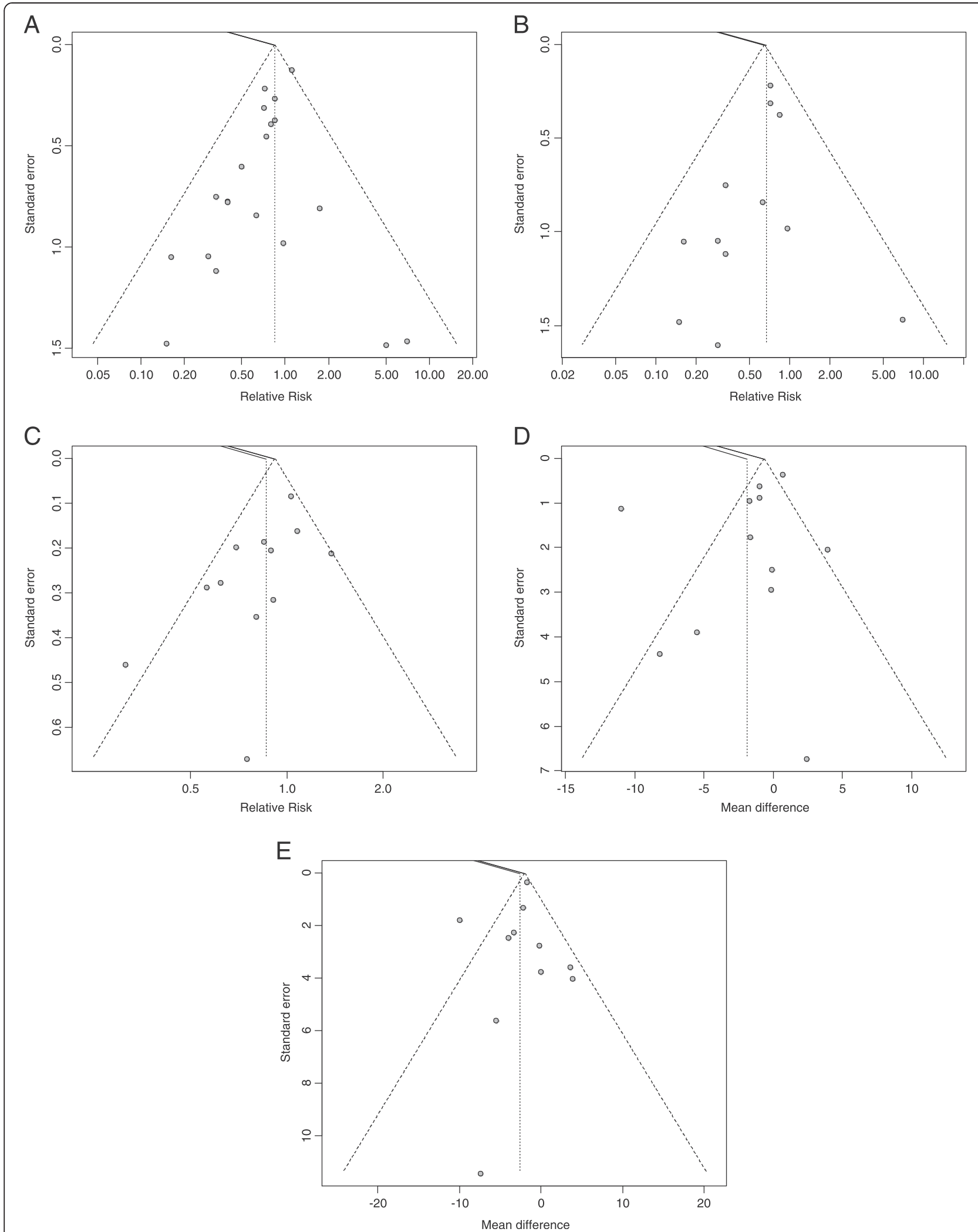

Figure 7 Funnel plots of primary and secondary outcomes. (A) Overall mortality: (Test for asymmetry $p=0.57$ ); (B) hospital mortality: (Test for asymmetry $\mathrm{p}=0.86$ ); (C) infection: (Test for asymmetry $\mathrm{p}=0.05$ ); (D) ICU LOS: (Test for asymmetry $\mathrm{p}=0.87$ ); (E) hospital LOS: (Test for asymmetry $\mathrm{p}=0.69$ ). LOS, length of stay. 
Overall, our results reveal that GLN supplementation continues to show a significant reduction in hospital mortality. In addition, GLN supplementation of complete nutrition was found to be associated with a strong trend towards a reduction in infectious complications, ICU LOS and a significant reduction in hospital LOS. GLN supplementation as part of nutrition support also continues to show a trend towards reduction of overall mortality in all trials examining both PN and EN nutrition delivery. The signal may be stronger for trials that utilize GLN supplementation as part of PN nutrition delivery. Importantly, no statistically significant signal of publication bias was observed in any of our tests for asymmetry as shown in the funnel plots in Figure 7.

A number of high-quality multi-center trials of parenteral GLN supplementation have been published recently, driving the need for this new critical care focused systematic review. A number of these key trials have strengths and weaknesses that are in some cases not reflected in the trial quality scoring utilized by this and other systematic reviews. Thus, a brief discussion of some of these key trials is useful. In short, these trials include the Scandinavian GLN trial [39], which examined 413 patients in multiple centers and demonstrated a benefit on ICU survival rates with parenteral GLN. A limitation of this trial was that it was stopped prior to achievement of its predefined enrollment goal due to slow enrollment. Another multi-center trial, the SIGNET trial, showed no benefit of short-term (approximately $\leq 4$ days), low-dose GLN (approximately $0.2 \mathrm{~g} / \mathrm{kg} / \mathrm{d}$ ) given parenterally to ICU patients on parenteral feeding [20]. Some concerns with this trial included lack of availability of some key data including issues regarding missing values, dropouts, protocol violations, and complete follow-up data. The actual values for GLN doses based on body weight have not been published and this has been viewed as a significant limitation of the trial. In an attempt to address the effect of quality of the trials and single-center versus multi-center trials included in the systematic review, we performed subgroup analyses to examine the role of these factors. We observed a more significant signal for parenteral GLN supplementation reducing mortality in the higher quality studies versus the lower quality trials. Single-center trials showed a greater benefit of GLN supplementation on mortality and overall infectious morbidity, although no signals of harm were observed in the multi-center trials of GLN on mortality. Interestingly, the beneficial effect of GLN on VAP was greater in the multi-center trials then the single-center trials. This is an endpoint that may deserve more focused examination in future trials of GLN supplementation in the ICU.

A number of other systematic reviews of GLN supplementation in either much broader populations, including noncritically ill patients, or specific populations (pancreatitis, burn injury, or elective surgery) have been published recently [11-14]. To our knowledge, our systematic analysis is the first to focus on the parenteral use of GLN supplementation of standard nutrition support in which all subjects are critically ill. When compared to the largest recent meta-analysis in a broader mixed elective surgery and critical care population many similarities with our data are observed [11]. First, with regard to mortality this recent analysis of GLN showed a very similar trend towards reduction of mortality $(\mathrm{RR}=0.89 ; 95 \% \mathrm{CI}, 0.77$ to 1.04 versus RR $0.88,95 \%$ CI $0.75,1.03$, in our analysis) in a patient population with a lower illness severity (where an effect on mortality would be less likely to be observed). No analysis of hospital mortality was reported in this previous analysis. With regard to infectious morbidity, this earlier meta-analysis showed a stronger statistically significant reduction of infectious morbidity $(\mathrm{RR}=0.83$; 95\% CI, 0.72 to 0.95 ) compared to our data where a nonsignificant trend of similar magnitude for a benefit on infection was observed (RR 0.86, 95\% CI 0.73, 1.02). Both the previous and current systematic reviews reported significant reductions on hospital LOSs of approximately 2.5 days (-2.35; $95 \%,-3.68$ to -1.02 versus $-2.56,95 \% \mathrm{CI}-4.71,-0.42$ in our analysis). Other key differences of our analysis versus the earlier report was that no direct industry funding was involved in support of our analysis (partial funding by an unrestricted industry grant was used to support this previous analysis), we included all of the world literature (including non-English trials) in our search, and we successfully attempted in all cases to contact the original authors when any questions of missing data or actual ICU patient inclusion was in question. For the first time we also examined the effect of GLN supplementation on VAP. We were also able to incorporate original source data from the unpublished multi-center American 'GLND' trial of GLN-supplementation of PN published in abstract form [44]. Finally, we performed funnel plots for all primary and key secondary endpoints examined by this analysis to examine possible publication bias associated with these endpoints.

The strength of our meta-analysis includes the use of several methods to reduce bias (comprehensive literature search, duplicate data abstraction, specific criteria for searching and analysis) and focus on clinically important primary outcomes. Notwithstanding, we are aware that our meta-analysis has several limitations. The major limitation is the small number of trials included in certain subgroup analyses such as $\mathrm{PN}$-supplementation of EN. We also unfortunately could not perform subgroup analysis for all endpoints due to limited numbers of trials examining the particular endpoints. Another potential weakness of any systematic review of randomized controlled nutrition trials has been pointed out by Vincent et al. recently [48]. This is the potential inability 
of a controlled nutrition trial to recreate 'real-life' patient conditions in the controlled setting of a trial (that is due to many patient exclusions). That said, we feel this systematic review best reflects all existing data in a wide variety of critical care settings of parenteral GLN supplementation to provide clinicians with most complete data to assist in making clinical decisions. Further, despite our attempts to be fully comprehensive in our search for all available trials we may not have been to include all available trials.

In spite of these limitations, we have demonstrated that traditional GLN supplementation used in the context of standard (predominantly parenteral) nutrition support in the critically ill may significantly reduce hospital mortality and shorten hospital LOS with a trend towards reduction in overall mortality and infectious complications, including VAP, and ICU LOS. Nonetheless, many questions on the ideal dose and timing of GLN supplementation in the ICU still remain unanswered. Further research is warranted to define the optimal dose and timing of supplementation of GLN in patients receiving full nutrition support. Recent data from REDOXS and other trials suggest that parenteral GLN should not be given in patients early in the acute phase of critical illness, in patients with multiple organ failure or in patients with unresuscitated shock requiring significant vasopressor support. Finally, based on the results of the REDOXS trial [6], we believe that high-dose parenteral or parenteral + enteral GLN (doses $>0.5 \mathrm{~g} / \mathrm{kg} / \mathrm{d}$ ) should not be used during the acute phase of critical illness.

\section{Conclusions}

In this comprehensive systematic review, we demonstrate that traditional parenteral GLN supplementation as a component of nutrition support (primarily added to $\mathrm{PN}$ ) is associated with a significant decrease in hospital mortality and length of hospital stay. GLN supplementation is also associated with trends towards reduced overall mortality, infectious complications and ICU LOS in critically ill patients. The therapeutic effect may be dependent on GLN dose given, with optimal benefit traditionally observed between 0.3 and $0.5 \mathrm{~g} / \mathrm{kg} / \mathrm{d}$. Thus, we recommend that parenteral GLN supplementation as a component of nutrition support be considered as an approach to improve outcomes of critical illness in selected patients. Our data here suggest that parenteral GLN supplementation, as a component of complete PN and/or EN support, is safe when administered following resolution of shock and multi-organ failure, and with daily doses less the $0.5 \mathrm{~g} / \mathrm{kg} / \mathrm{d}$. Focused clinical trials on the clinical efficacy of parenteral GLN supplementation combined with adequate and complete specialized nutrition support are needed in critically ill patients at risk of GLN depletion who do not have multiple organ failure or ongoing shock.

\section{Key messages}

- Critical illness is characterized by severe metabolic stress and glutamine depletion has been associated with increased mortality in some recent studies. In this context, supplementation of parenteral glutamine, predominantly as a component of parenteral nutrition, may improve clinical outcomes when given to appropriate patients as part of complete nutrition support.

- Supplemental parenteral glutamine may significantly decrease hospital mortality and shorten hospital LOS in critically ill patients.

- Supplemental parenteral glutamine given as a component of nutrition support is associated with a trend towards a reduction in overall mortality, infectious morbidity, and ICU LOS in critically ill patients.

- We suggest supplemental glutamine should not be given in a high dose $(>0.5 \mathrm{~g} / \mathrm{kg} /$ day $)$ or in patients early in the acute phase of critical illness in patients with multiple organ failure or unresuscitated shock requiring significant vasopressor support.

- When parenteral nutrition is prescribed to critically ill patients, parenteral supplementation with glutamine should continue to be considered safe and may potentially improve outcomes in the ICU in patients without specific contraindications.

\section{Additional file}

Additional file 1: Table S1. Studies of glutamine supplementation in patients not included in the analysis.

\section{Abbreviations}

Cl: confidence interval; EN: enteral nutrition; GLN: glutamine; ICU: intensive care unit; ITT: intention to treat; IV: intravenous; LOS: length of stay; PN: parenteral nutrition; RR: relative risk; VAP: ventilator-associated pneumonia; WMD: weighted mean difference.

\section{Competing interests}

No funding for the development, writing, or submission of this manuscript was received. Paul Wischmeyer has research funding for the REDOXS trial of glutamine and antioxidants from Fresenius Kabi. Rupinder Dhaliwal has no conflict of interest to declare. Michele McCall has no conflict of interest to declare. Thomas R. Ziegler has research funding for the GLND trial of glutamine-supplemented total parenteral nutrition in surgical critical care from Fresenius Kabi. Daren K. Heyland has research funding for the REDOXS trial of glutamine and antioxidants from Fresenius Kabi.

\section{Authors' contributions}

PW contributed to development of the manuscript concept, study grading, study selection, evaluation, interpretation of data and performed the primary authoring and editing of the manuscript. RD contributed to development of the manuscript concept, study grading selection, evaluation, interpretation of data and performed much of the primary statistical analysis and meta-analysis data analysis. He also performed significant work authoring and editing all drafts of the manuscript. MM contributed to development of the manuscript concept, study grading, study selection, evaluation, interpretation of data and assisted in editing the manuscript. TZ assisted in 
editing the manuscript drafts and confirmed the primary data of the GLND trial. DH contributed to development of the manuscript concept, study grading, study selection, evaluation, interpretation of data and assisted in the primary editing of all drafts of the manuscript. All authors read and approved the final manuscript.

\section{Acknowledgements}

The authors wish to acknowledge Xuran Jiang (a biostatistician) for assistance with statistical analysis and figure generation for this manuscript.

\section{Author details}

${ }^{1}$ Department of Anesthesiology, University of Colorado School of Medicine, 12700 East 19th Avenue, Aurora, CO 80045, USA. ${ }^{2}$ Clinical Evaluation Research Unit, Kingston General Hospital, 76 Stuart Street, Kingston, ON K7L 2V7, Canada. ${ }^{3}$ Medical/Surgical Intensive Care Unit, St. Michael's Hospital, 30 Bond Street, Toronto, ON M5B 1W8, Canada. ${ }^{4}$ Department of Medicine, Center for Clinical and Molecular Nutrition, Emory University School of Medicine, 1648 Pierce Drive North East, Atlanta, GA 30322, USA. ${ }^{5}$ Department of Medicine, Queens University, Kingston, Ontario, Canada.

Received: 21 October 2013 Accepted: 14 March 2014

Published: 18 April 2014

\section{References}

1. Wischmeyer PE: Glutamine: role in critical illness and ongoing clinical trials. Curr Opin Gastroenterol 2008, 24:190-197.

2. Coeffier $M$, Dechelotte $P$ : The role of glutamine in intensive care unit patients: mechanisms of action and clinical outcome. Nutr Rev 2005, 63:65-69.

3. Gamrin L, Essen P, Forsberg AM, Hultman E, Wernerman J: A descriptive study of skeletal muscle metabolism in critically ill patients: free amino acids, energy-rich phosphates, protein, nucleic acids, fat, water, and electrolytes. Crit Care Med 1996, 24:575-583.

4. Newsholme EA, Crabtree B, Ardawi MS: Glutamine metabolism in lymphocytes: its biochemical, physiological and clinical importance. Q J Exp Physiol 1985, 70:473-489.

5. Wilmore DW: The effect of glutamine supplementation in patients following elective surgery and accidental injury. J Nutr 2001, 131:2543S-2549S. discussion 2550S-2541S.

6. Heyland D, Muscedere J, Wischmeyer PE, Cook D, Jones G, Albert M, Elke G, Berger MM, Day AG, Canadian Critical Care Trials G: A randomized trial of glutamine and antioxidants in critically ill patients. N Engl J Med 2013, 368:1489-1497.

7. Oudemans-van Straaten HM, Bosman RJ, Treskes M, van der Spoel HJ, Zandstra DF: Plasma glutamine depletion and patient outcome in acute ICU admissions. Intensive Care Med 2001, 27:84-90.

8. Rodas PC, Rooyackers $\mathrm{O}$, Hebert C, Norberg A, Wernerman J: Glutamine and glutathione at ICU admission in relation to outcome. Clin Sci 2012, 122:591-597.

9. Dechelotte $P$, Hasselmann M, Cynober L, Allaouchiche B, Coeffier M, Hecketsweiler B, Merle V, Mazerolles M, Samba D, Guillou YM, Petit J, Mansoor O, Colas G, Cohendy R, Barnoud D, Czernichow P, Bleichner G: L-alanyl-L-glutamine dipeptide-supplemented total parenteral nutrition reduces infectious complications and glucose intolerance in critically ill patients: the French controlled, randomized, double-blind, multicenter study. Crit Care Med 2006, 34:598-604.

10. Goeters C, Wenn A, Mertes N, Wempe C, Van Aken H, Stehle P, Bone HG: Parenteral L-alanyl-L-glutamine improves 6-month outcome in critically ill patients. Crit Care Med 2002, 30:2032-2037.

11. Bollhalder L, Pfeil AM, Tomonaga Y, Schwenkglenks M: A systematic literature review and meta-analysis of randomized clinical trials of parenteral glutamine supplementation. Clin Nutr 2013, 32:213-223.

12. Lin JJ, Chung XJ, Yang CY, Lau HL: A meta-analysis of trials using the intention to treat principle for glutamine supplementation in critically ill patients with burn. Burns 2013, 39:565-570.

13. Zheng YM, Li F, Zhang MM, Wu XT: Glutamine dipeptide for parenteral nutrition in abdominal surgery: a meta-analysis of randomized controlled trials. World J Gastroenterol 2006, 12:7537-7541.

14. Zhong Z, Liang C, Gong S: Intravenous glutamine for sever acute pancreatitis: a meta analysis. World J Crit Care Med 2013, 2:4-8.
15. Duska F, Fric M, Waldauf P, Pazout J, Andel M, Mokrejs P, Tuma P, Pachl J: Frequent intravenous pulses of growth hormone together with glutamine supplementation in prolonged critical illness after multiple trauma: effects on nitrogen balance, insulin resistance, and substrate oxidation. Crit Care Med 2008, 36:1707-1713.

16. Bellomo R, Warrillow SJ, Reade MC: Why we should be wary of single-center trials. Crit Care Med 2009, 37:3114-3119.

17. Review Manager (RevMan) [Computer program]. Version 5.2. Copenhagen: The Nordic Cochrane Centre, The Cochrane Collaboration, 2012.

18. Higgins JP, Thompson SG: Quantifying heterogeneity in a meta-analysis. Stat Med 2002, 21:1539-1558.

19. Rucker G, Schwarzer G, Carpenter J: Arcsine test for publication bias in meta-analyses with binary outcomes. Stat Med 2008, 27:746-763.

20. Andrews PJ, Avenell A, Noble DW, Campbell MK, Croal BL, Simpson WG, Vale LD, Battison CG, Jenkinson DJ, Cook JA: Randomised trial of glutamine, selenium, or both, to supplement parenteral nutrition for critically ill patients. BMJ 2011, 342:d1542.

21. Cai G, Yan J, Zhang Z, Yu Y: Immunomodulatory effects of glutamineenriched nutritional support in elderly patients with severe sepsis: a prospective, randomized, controlled study. J Organ Dysfunct 2008, 4:31-37.

22. Cekmen N, Aydin A, Erdemli O: The impact of L-alanyl-L-glutamine dipeptide supplemented total parenteral nutrition on clinical outcome in critically patients. E-SPEN, Euro e-J Clin Nutr Metab 2011, 6:64-67.

23. Eroglu $A$ : The effect of intravenous alanyl-glutamine supplementation on plasma glutathione levels in intensive care unit trauma patients receiving enteral nutrition: the results of a randomized controlled trial. Anesth Analg 2009, 109:502-505.

24. Estivariz CF, Griffith DP, Luo M, Szeszycki EE, Bazargan N, Dave N, Daignault NM, Bergman GF, McNally T, Battey CH, Furr CE, Hao L, Ramsay JG, Accardi CR, Cotsonis GA, Jones DP, Galloway JR, Ziegler TR: Efficacy of parenteral nutrition supplemented with glutamine dipeptide to decrease hospital infections in critically ill surgical patients. JPEN J Parenter Enteral Nutr 2008, 32:389-402

25. Fuentes-Orozco C, Anaya-Prado R, Gonzalez-Ojeda A, Arenas-Marquez H, Cabrera-Pivaral C, Cervantes-Guevara G, Barrera-Zepeda LM: L-alanyl-Lglutamine-supplemented parenteral nutrition improves infectious morbidity in secondary peritonitis. Clin Nutr 2004, 23:13-21.

26. Fuentes-Orozco C, Cervantes-Guevara G, Mucino-Hernandez I, Lopez-Ortega A, Ambriz-Gonzalez G, Gutierrez-de-la-Rosa JL, Gomez-Herrera E, HermosilloSandoval JM, Gonzalez-Ojeda A: L-alanyl-L-glutamine-supplemented parenteral nutrition decreases infectious morbidity rate in patients with severe acute pancreatitis. JPEN J Parenter Enteral Nutr 2008, 32:403-411.

27. Grau T, Bonet A, Minambres E, Pineiro L, Irles JA, Robles A, Acosta J, Herrero I, Palacios V, Lopez J, Blesa A, Martinez P: The effect of L-alanylL-glutamine dipeptide supplemented total parenteral nutrition on infectious morbidity and insulin sensitivity in critically ill patients. Crit Care Med 2011, 39:1263-1268.

28. Griffiths RD, Allen KD, Andrews FJ, Jones C: Infection, multiple organ failure, and survival in the intensive care unit: influence of glutaminesupplemented parenteral nutrition on acquired infection. Nutrition 2002, 18:546-552

29. Griffiths RD, Jones C, Palmer TE: Six-month outcome of critically ill patients given glutamine-supplemented parenteral nutrition. Nutrition 1997, 13:295-302.

30. He Xian-li MQJ, Lu JG, Chu YK, Du XL: Effect of total parenteral nutrition (TPN) with and without glutamine dipeptide supplementation on outcome in severe acute pancreatitis (SAP). Clin Nutr Suppl 2004, 1:43-47.

31. Luo M, Bazargan N, Griffith DP, Estivariz CF, Leader LM, Easley KA, Daignault NM, Hao L, Meddings JB, Galloway JR, Blumberg JB, Jones DP, Ziegler TR: Metabolic effects of enteral versus parenteral alanyl-glutamine dipeptide administration in critically ill patients receiving enteral feeding: a pilot study. Clin Nutr 2008, 27:297-306.

32. Ozgultekin A, Turan G, Durmus Y, Dincer E, Akgun N: Comparison of the efficacy of parenteral and branched-chain amino acid solutions given as extra supplements in parallel to the enteral nutrition in head trauma. E-SPEN, Euro e-J of Clin Nutr Metab 2008, 3:211-216.

33. Palmese S, Odierna I, Scarano D, Scibili AC, Natale A, Pezza M: Early enteral nutrition enriched with FOS and intravenous glutamine supplementation in intensive care unit patients. Nutr Ther Metab 2006, 24:140-146. 
34. Perez-Barcena J, Crespi C, Regueiro V, Marse P, Raurich JM, Ibanez J, Bengoechea JA, Garcia De Lorenzo-Mateos A: Lack of effect of glutamine administration to boost the innate immune system response in trauma patients in the intensive care unit. Crit Care 2010, 14:R233.

35. Perez-Barcena J, Regueiro V, Marse P, Raurich JM, Rodriguez A, Ibanez J, de Lorenzo Mateos AG, Bengoechea JA: Glutamine as a modulator of the immune system of critical care patients: effect on toll-like receptor expression. A preliminary study. Nutrition 2008, 24:522-527.

36. Powell-Tuck J, Jamieson CP, Bettany GE, Obeid O, Fawcett HV, Archer C, Murphy DL: A double blind, randomised, controlled trial of glutamine supplementation in parenteral nutrition. Gut 1999, 45:82-88.

37. Sahin H, Mercanligil SM, Inanc N, Ok E: Effects of glutamine-enriched total parenteral nutrition on acute pancreatitis. Eur J Clin Nutr 2007, 61:1429-1434

38. Tian H, Wang KF, Wu TJ: Effect of total parenteral nutrition with supplementation of glutamine on the plasma diamine oxidase activity and D-lactate content in patients with multiple organ dysfunction syndrome. Zhongguo Wei Zhong Bing Ji Jiu Yi Xue 2006, 18:616-618.

39. Wernerman J, Kirketeig T, Andersson B, Berthelson $H$, Ersson A, Friberg $H$, Guttormsen AB, Hendrikx S, Pettila V, Rossi P, Sjoberg F, Winso O: Scandinavian glutamine trial: a pragmatic multi-centre randomised clinical trial of intensive care unit patients. Acta Anaesthesiol Scand 2011 55:812-818.

40. Wischmeyer PE, Lynch J, Liedel J, Wolfson R, Riehm J, Gottlieb L, Kahana M Glutamine administration reduces gram-negative bacteremia in severely burned patients: a prospective, randomized, double-blind trial versus isonitrogenous control. Crit Care Med 2001, 29:2075-2080.

41. Yang SQ, Xu JG: Effect of glutamine on serum interleukin-8 and tumor necrosis factor-alpha levels in patients with severe pancreatitis. Nan Fang Yi Ke Da Xue Xue Bao 2008, 28:129-131.

42. Ye-Ping Zhou Z-MJ, Yong-Hua S, Gui-Zhen H, Hong S: The effects of supplemental glutamine dipeptide on gut integrity and clinical outcome after major escharectomy in severe burns: a randomized, double-blind, controlled clinical trial. Clin Nutr Supp/ 2004, 1:55-60.

43. Zhang Z, Qin HD, Ni HB, Xu Y, Wu HR, Cheng H, Wang SK: Effect of early enriched parenteral alanyl-glutamine on heat shock protein 70 (HSP70) expression in critical patients. Zhongguo Wei Zhong Bing Ji Jiu Yi Xue 2007, 19:481-484

44. Ziegler T, May A, Hebbar G, Kudsk K, Sax H, Blumberg H, Easley K, Wischmeyer P: Glutamine dipeptide-supplemented parenteral nutrition in surgical ICU patients: Results of an American randomized, double-blind, multicenter trial. Clin Nutr Supp/ 2012, 7:265

45. Novak F, Heyland DK, Avenell A, Drover JW, Su X: Glutamine supplementation in serious illness: a systematic review of the evidence. Crit Care Med 2002, 30:2022-2029.

46. Bongers T, Griffiths RD, McArdle A: Exogenous glutamine: the clinical evidence. Crit Care Med 2007, 35:S545-S552.

47. Critical care nutrition website. [http://www.criticalcarenutrition.com]

48. Vincent $J$, Preiser JC: Are prospective cohort studies an appropriate tool to answer clinical nutrition questions? Curr Opin Clin Nutr Metab Care 2013, 16:182-186

doi: $10.1186 /$ cc13836

Cite this article as: Wischmeyer et al:: Parenteral glutamine supplementation in critical illness: a systematic review. Critical Care 2014 18:R76

\section{Submit your next manuscript to BioMed Central and take full advantage of:}

- Convenient online submission

- Thorough peer review

- No space constraints or color figure charges

- Immediate publication on acceptance

- Inclusion in PubMed, CAS, Scopus and Google Scholar

- Research which is freely available for redistribution 